\title{
CHEMICAL REACTION SYSTEMS WITH TORIC STEADY STATES
}

\author{
MERCEDES PÉREZ MILLÁN, ALICIA DICKENSTEIN, ANNE SHIU, AND CARSTEN CONRADI
}

\begin{abstract}
Mass-action chemical reaction systems are frequently used in Computational Biology. The corresponding polynomial dynamical systems are often large (consisting of tens or even hundreds of ordinary differential equations) and poorly parametrized (due to noisy measurement data and a small number of data points and repetitions). Therefore, it is often difficult to establish the existence of (positive) steady states or to determine whether more complicated phenomena such as multistationarity exist. If, however, the steady state ideal of the system is a binomial ideal, then we show that these questions can be answered easily. The focus of this work is on systems with this property, and we say that such systems have toric steady states. Our main result gives sufficient conditions for a chemical reaction system to have toric steady states. Furthermore, we analyze the capacity of such a system to exhibit positive steady states and multistationarity. Examples of systems with toric steady states include weakly-reversible zero-deficiency chemical reaction systems. An important application of our work concerns the networks that describe the multisite phosphorylation of a protein by a kinase/phosphatase pair in a sequential and distributive mechanism.
\end{abstract}

Keywords: chemical reaction networks, mass-action kinetics, multistationarity, multisite phosphorylation, binomial ideal.

\section{INTRODUCTION}

Ordinary differential equations (ODEs) are an important modeling tool in Systems Biology and many other areas of Computational Biology. Due to the inherent complexity of biological systems, realistic models are often large, both in terms of the number of states and the (unknown) parameters. Moreover, models are often poorly parametrized, a consequence of noisy measurement data, a small number of data points, and a limited number of repetitions. Hence, for mass-action chemical reaction systems, the focus of the present article, simply establishing the existence of (positive) steady states can be demanding, as it requires the solution of a large polynomial system with unknown coefficients (usually the parameters). Moreover, due to the predominant parameter uncertainty, one is often not interested in establishing the existence of a particular steady state, but rather in obtaining a parametrization of all steady states - preferably in terms of the system parameters [34]. Frequently one is also interested in the existence of multiple steady states (multistationarity), for example, in modeling the cell cycle [2, 3, 28, signal transduction [23, 26] or cellular differentiation [32, 33. For general polynomial systems with unknown coefficients, the tasks of obtaining positive solutions or a parametrization of positive solutions, and deciding about multiple positive solutions, are clearly challenging. For the systems considered in this article chemical reaction systems with toric steady states - these questions can be answered easily.

We say that a polynomial dynamical system $d x / d t=f(x)$ has toric steady states if the ideal generated by its steady state equations is a binomial ideal (see Definition 2.2). We give sufficient conditions for a chemical reaction system to have toric steady states (Theorems 3.8 and 3.19) and show in this case that the steady state locus has a nice monomial parametrization (Theorems 3.11 and 3.201). Furthermore, we show that the existence of positive steady states in this case is straightforward to check (Theorem [5.5).

There are several important classes of mass-action kinetics chemical reaction systems which have toric steady states. These include usual instances of detailed-balanced systems in the sense of

\footnotetext{
${ }^{1} \mathrm{MPM}$ and AD were partially supported by UBACYT X064, CONICET PIP 112-200801-00483, and ANPCyT PICT 2008-0902, Argentina. AS was supported by the NSF (DMS-1004380). CC was supported by ForSys/MaCS 0313922 .
} 
Feinberg, Horn, and Jackson [11, 12, 20, 21, which show particularly nice dynamical behavior. These systems are weakly-reversible, a hypothesis we do not impose here.

A chemical reaction system with toric steady states of great biological importance is the multisite phosphorylation system; this network describes the $n$-site phosphorylation of a protein by a kinase/phosphatase pair in a sequential and distributive mechanism. Biochemically, these systems play an important role in signal transduction networks, cell cycle control, or cellular differentiation: for example, members of the family of mitogen-activated kinase cascades consist of several such phosphorylation systems with $n=2$ or $n=3$ (see e.g. [22, 29]), the progression from G1 to S phase in the cell cycle of budding yeast is controlled by a system with $n=9$ (by way of the protein Sic1, see e.g. [9]), and a system with $n=13$ plays an important role in T-cell differentiation (by way of the protein NFAT [17, 18, 24]).

Consequently there exists a body of work on the mathematics of phosphorylation systems and the more general class of post-translational modification systems: for example, Conradi et al. [6], Wang and Sontag [36], Manrai and Gunawardena [25], and Thomson and Gunawardena [34, 35]. While the first two references are concerned with the number of steady states and multistationarity, the references of Gunawardena et al. deal with parametrizing all positive steady states. The present article builds on these earlier results. In fact, the family of monomial parametrizations obtained here for multisite phosphorylation systems (Theorem 4.3) is a specific instance of a rational parametrization theorem due to Thomson and Gunawardena, and one parametrization of the family was analyzed earlier by Wang and Sontag. Furthermore, we show that by using results from [6] one can determine whether multistationarity exists for systems with toric steady states by analyzing certain linear inequality systems. In this sense our results can be seen as a generalization of [6].

This article is organized as follows. Section 2 provides an introduction to the mathematics of chemical reaction systems. Our main results on toric steady states appear in Section 3 Theorems 3.8 and 3.19 give sufficient criteria for a system to exhibit toric steady states, and Theorems 3.11 and 3.20 give parametrizations for the steady state locus. As an application of this work, we analyze the steady state loci of multisite phosphorylation systems in Section 4. Theorem 4.3 summarizes our results: we show that these systems have toric steady states for any choice of reaction rate constants, and we give an explicit parametrization of the steady state locus. Section 5 focuses on multiple steady states for chemical reaction systems with toric steady states. Theorem 5.5 gives a criterion for such a system to exhibit multistationarity, and we make the connection to a related criterion due to Feinberg.

\section{Chemical Reaction network theory}

In this section we recall the basic setup of chemical reaction systems, and we introduce in $\S 2.2$ the precise definition of systems with toric steady states. We first present an intuitive example that illustrates how a chemical reaction network gives rise to a dynamical system. An example of a chemical reaction, as it usually appears in the literature, is the following:

$$
A+B \stackrel{\kappa}{\longrightarrow} 3 A+C
$$

In this reaction, one unit of chemical species $A$ and one of $B$ react (at reaction rate $\kappa$ ) to form three units of $A$ and one of $C$. The educt (or reactant or source) $A+B$ and the product $3 A+C$ are called complexes. We will refer to complexes such as $A+B$ that are the educt of a reaction as educt complexes. The concentrations of the three species, denoted by $x_{A}, x_{B}$, and $x_{C}$, will change in time as the reaction occurs. Under the assumption of mass-action kinetics, species $A$ and $B$ react at a rate proportional to the product of their concentrations, where the proportionality constant is the rate constant $\kappa$. Noting that the reaction yields a net change of two units in the amount of $A$, we 
obtain the first differential equation in the following system:

$$
\begin{aligned}
& \frac{d}{d t} x_{A}=2 \kappa x_{A} x_{B}, \\
& \frac{d}{d t} x_{B}=-\kappa x_{A} x_{B}, \\
& \frac{d}{d t} x_{C}=\kappa x_{A} x_{B} .
\end{aligned}
$$

The other two equations arise similarly. A chemical reaction network consists of finitely many reactions. The differential equations that a network defines are comprised of a sum of the monomial contribution from the reactant of each chemical reaction in the network; these differential equations will be defined in equation (2.3).

2.1. Chemical reaction systems. We now provide precise definitions. A chemical reaction network is a finite directed graph whose vertices are labeled by complexes and whose edges are labeled by parameters (reaction rate constants). Specifically, the digraph is denoted $G=(V, E)$, with vertex set $V=\{1,2, \ldots, m\}$ and edge set $E \subseteq\{(i, j) \in V \times V: i \neq j\}$. Throughout this article, the integer unknowns $m, s$, and $r$ denote the numbers of complexes, species, and edges (reactions), respectively. Linkage classes refer to the connected components of a network, and terminal strong linkage classes refer to the maximal strongly connected subgraphs in which there are no edges (reactions) from a complex in the subgraph to a complex outside the subgraph. The vertex $i$ of $G$ represents the $i$-th chemical complex, and we associate to it the monomial

$$
x^{y_{i}}=x_{1}^{y_{i 1}} x_{2}^{y_{i 2}} \cdots x_{s}^{y_{i s}} .
$$

More precisely, if the $i$-th complex is $y_{i 1} A+y_{i 2} B+\cdots$ (where $y_{i j} \in \mathbb{Z}_{\geq 0}$ for $j=1,2, \ldots, s$ ), then it defines the monomial $x_{A}^{y_{i 1}} x_{B}^{y_{i 2}} \cdots$. For example, the two complexes in the network (2.1) give rise to the monomials $x_{A} x_{B}$ and $x_{A}^{3} x_{C}$, which determine two vectors $y_{1}=(1,1,0)$ and $y_{2}=(3,0,1)$. These vectors define the rows of an $m \times s$-matrix of non-negative integers, which we denote by $Y=\left(y_{i j}\right)$. Next, the unknowns $x_{1}, x_{2}, \ldots, x_{s}$ represent the concentrations of the $s$ species in the network, and we regard them as functions $x_{i}(t)$ of time $t$. The monomial labels form the entries in the following vector:

$$
\Psi(x)=\left(x^{y_{1}}, x^{y_{2}}, \ldots, x^{y_{m}}\right)^{t} .
$$

A directed edge $(i, j) \in E$ represents a reaction from the $i$-th chemical complex to the $j$-th chemical complex. Each edge is labeled by a positive parameter $\kappa_{i j}$ which represents the rate constant of the reaction. In this article, we will treat the rate constants $\kappa_{i j}$ as unknowns; we are interested in the family of dynamical systems that arise from a given network as the rate constants $\kappa_{i j}$ vary.

The main application of our results are chemical reaction networks under mass-action kinetics. Therefore, even if the principal results in $\S 3$ hold for general polynomial dynamical systems, we assume in what follows mass-action kinetics. We now explain how mass-action kinetics defines a dynamical system from a chemical reaction network. Let $A_{\kappa}$ denote the negative of the Laplacian of the chemical reaction network $G$. In other words, $A_{\kappa}$ is the $m \times m$-matrix whose off-diagonal entries are the $\kappa_{i j}$ and whose row sums are zero. Now we define the complex-to-species rate matrix of size $s \times m$ to be

$$
\Sigma:=Y^{t} \cdot A_{\kappa}^{t}
$$

The reaction network $G$ defines the following dynamical system:

$$
\frac{d x}{d t}=\left(\frac{d x_{1}}{d t}, \frac{d x_{2}}{d t}, \ldots, \frac{d x_{s}}{d t}\right)^{t}=\Sigma \cdot \Psi(x) .
$$


We see that the right-hand side of each differential equation $d x_{l} / d t$ is a polynomial in the polynomial $\operatorname{ring} \mathbb{R}\left[\left(\kappa_{i j}\right)_{(i, j) \in E}, x_{1}, x_{2}, \ldots, x_{s}\right]$. A chemical reaction system refers to the dynamical system (2.3) arising from a specific chemical reaction network $G$ and a choice of rate parameters $\left(\kappa_{i j}^{*}\right) \in \mathbb{R}_{>0}^{r}$ (recall that $r$ denotes the number of reactions).

Example 2.1. The following chemical reaction network is the 1-site phosphorylation system:

$$
\begin{aligned}
S_{0}+E \underset{k_{\mathrm{off}_{0}}}{\stackrel{k_{\mathrm{on}_{0}}}{\rightleftarrows}} E S_{0} \stackrel{k_{\mathrm{cat}_{0}}}{\rightarrow} S_{1}+E \\
S_{1}+F \underset{l_{\mathrm{off}_{0}}}{\stackrel{l_{\mathrm{on}_{0}}}{\rightleftarrows}} F S_{1} \stackrel{l_{\text {cat }}}{\rightarrow} S_{0}+F .
\end{aligned}
$$

The key players in this network are a kinase enzyme (E), a phosphatase enzyme $(F)$, and two substrates $\left(S_{0}\right.$ and $\left.S_{1}\right)$. The substrate $S_{1}$ is obtained from the unphosphorylated protein $S_{0}$ by attaching a phosphate group to it via an enzymatic reaction involving $E$. Conversely, a reaction involving $F$ removes the phosphate group from $S_{1}$ to obtain $S_{0}$. The intermediate complexes $E S_{0}$ and $E S_{1}$ are the bound enzyme-substrate complexes. Under the ordering of the 6 species as $\left(S_{0}, S_{1}, E S_{0}, F S_{1}, E, F\right)$ and the 6 complexes as $\left(S_{0}+E, S_{1}+E, E S_{0}, S_{0}+F, S_{1}+F, F S_{1}\right)$, the matrices whose product defines the dynamical system (2.3) follow:

$$
\begin{gathered}
\Psi(x)=\left(x_{S_{0}} x_{E}, x_{S_{1}} x_{E}, x_{E S_{0}}, x_{S_{0}} x_{F}, x_{S_{1}} x_{F}, x_{F S_{1}}\right)^{t}=\left(x_{1} x_{5}, x_{2} x_{5}, x_{3}, x_{1} x_{6}, x_{2} x_{6}, x_{4}\right)^{t}, \\
Y^{t}=\left[\begin{array}{ccccccc}
1 & 0 & 0 & 1 & 0 & 0 \\
0 & 1 & 0 & 0 & 1 & 0 \\
0 & 0 & 1 & 0 & 0 & 0 \\
0 & 0 & 0 & 0 & 0 & 1 \\
1 & 1 & 0 & 0 & 0 & 0 \\
0 & 0 & 0 & 1 & 1 & 0
\end{array}\right], \text { and } \\
A_{\kappa}^{t}:=\left[\begin{array}{cccccc}
-k_{\mathrm{on}_{0}} & 0 & k_{\text {off }_{0}} & 0 & 0 & 0 \\
0 & 0 & k_{\text {cat }} & 0 & 0 & 0 \\
k_{\mathrm{on}_{0}} & 0 & -k_{\text {off }_{0}}-k_{\text {cat }_{0}} & 0 & 0 & 0 \\
0 & 0 & 0 & 0 & 0 & l_{\text {cat }_{0}} \\
0 & 0 & 0 & 0 & -l_{\text {on }_{0}} & l_{\text {off }_{0}} \\
0 & 0 & 0 & 0 & l_{\text {on }_{0}} & -l_{\text {cat }_{0}}-l_{\text {off }_{0}}
\end{array}\right] .
\end{gathered}
$$

We will study generalizations of this network in this article.

The stoichiometric subspace is the vector subspace spanned by the reaction vectors $y_{j}-y_{i}$ (where $(i, j)$ is an edge of $G)$, and we will denote this space by $\mathcal{S}$ :

$$
\mathcal{S}:=\mathbb{R}\left\{y_{j}-y_{i} \mid(i, j) \in E\right\} .
$$

In the earlier example shown in (2.1), we have $y_{2}-y_{1}=(2,-1,1)$, which means that with the occurrence of each reaction, two units of $A$ and one of $C$ are produced, while one unit of $B$ is consumed. This vector $(2,-1,1)$ spans the stoichiometric subspace $\mathcal{S}$ for the network (2.1). Note that the vector $\frac{d x}{d t}$ in (2.3) lies in $\mathcal{S}$ for all time $t$. In fact, a trajectory $x(t)$ beginning at a positive vector $x(0)=x^{0} \in \mathbb{R}_{>0}^{s}$ remains in the stoichiometric compatibility class (also called an "invariant polyhedron"), which we denote by

$$
\mathcal{P}_{x^{0}}:=\left(x^{0}+\mathcal{S}\right) \cap \mathbb{R}_{\geq 0}^{s},
$$

for all positive time. In other words, this set is forward-invariant with respect to the dynamics (2.3). It follows that any stoichiometric compatibility class of a network has the same dimension as the stoichiometric subspace. 
2.2. Steady states. We present the definition of systems with toric steady states. For background information on the algebraic tools we use, we refer the reader to the nice textbook of Cox, Little, and O'Shea [7].

Recall that an ideal in $\mathbb{R}\left[x_{1}, x_{2}, \ldots, x_{s}\right]$ is called a binomial ideal if it can be generated by binomials (i.e., polynomials with at most two terms). The basic building blocks of binomial ideals are the prime binomial ideals, which are called toric ideals [10].

Definition 2.2. Consider a polynomial dynamical system $d x_{i} / d t=f_{i}(x)$, for $i=1,2, \ldots, s$, with $f_{1}, f_{2}, \ldots, f_{s} \in \mathbb{R}\left[x_{1}, x_{2}, \ldots, x_{s}\right]$. We are interested in the real zeros of the steady state ideal:

$$
J_{\Sigma \Psi}=\left\langle f_{1}, f_{2}, \ldots, f_{s}\right\rangle=\left\{\sum_{i=1}^{s} h_{i}(x) f_{i}(x) \quad \mid \quad h_{i}(x) \in \mathbb{R}\left[x_{1}, x_{2}, \ldots, x_{s}\right] \text { for } 1 \leq i \leq s\right\} .
$$

The real zeros of $J_{\Sigma \Psi}$ are called steady states, and the term steady state locus is used to denote the set of real zeros of $J_{\Sigma \Psi}$ :

$$
\left\{x^{*} \in \mathbb{R}^{s} \quad \mid \quad f_{1}\left(x^{*}\right)=f_{2}\left(x^{*}\right)=\cdots=f_{s}\left(x^{*}\right)=0\right\} .
$$

We say that the polynomial dynamical system has toric steady states if $J_{\Sigma \Psi}$ is a binomial ideal and it admits real zeros.

We are interested in positive steady states $x \in \mathbb{R}_{>0}^{s}$ and will not be concerned with boundary steady states $x \in\left(\mathbb{R}_{\geq 0}^{s} \backslash \mathbb{R}_{>0}^{s}\right)$.

This article focuses on mass-action kinetics chemical reaction systems. In this case, the polynomials $f_{1}, f_{2}, \ldots, f_{s}$ correspond to the rows of the system (2.3). In general, having toric steady states depends both on the reaction network and on the particular rate constants, as the following simple example shows.

Example 2.3 (Triangle network). Let $s=2, m=3$, and let $G$ be the following network:

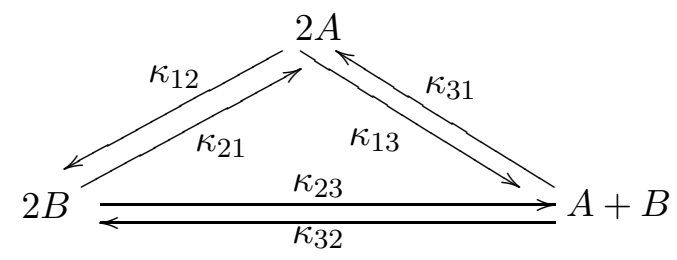

We label the three complexes as $x^{y_{1}}=x_{1}^{2}, x^{y_{2}}=x_{2}^{2}, x^{y_{3}}=x_{1} x_{2}$, and we define $\kappa_{i j}$ to be the (real positive) rate constant of the reaction from complex $x^{y_{i}}$ to complex $x^{y_{j}}$. The resulting mass-action kinetics system (2.3) equals

$$
\frac{d x_{1}}{d t}=-\frac{d x_{2}}{d t}=\left(-2 \kappa_{12}-\kappa_{13}\right) x_{1}^{2}+\left(2 \kappa_{21}+\kappa_{23}\right) x_{2}^{2}+\left(\kappa_{31}-\kappa_{32}\right) x_{1} x_{2} .
$$

Then, the steady state locus in $\mathbb{R}^{2}$ is defined by this single trinomial. As only the coefficient of $x_{1} x_{2}$ can be zero, this system has toric steady states if and only if $\kappa_{31}=\kappa_{32}$.

A chemical reaction system exhibits multistationarity if there exists a stoichiometric compatibility class $\mathcal{P}_{x^{0}}$ with two or more steady states in its relative interior. A system may admit multistationarity for all, some, or no choices of positive rate constants $\kappa_{i j}$; if such rate constants exist, then we say that the network has the capacity for multistationarity.

2.3. The deficiency of a chemical reaction network. The deficiency $\delta$ of a chemical reaction network is an important invariant. For a chemical reaction network, recall that $m$ denotes the number of complexes. Denote by $l$ the number of linkage classes. Most of the networks considered in this article have the property that each linkage class contains a unique terminal strong linkage 
class. In this case, Feinberg showed that the deficiency of the network can be computed in the following way:

$$
\delta:=m-l-\operatorname{dim}(\mathcal{S}),
$$

where $\mathcal{S}$ denotes the stoichiometric subspace. Note that in this case the deficiency depends only on the reaction network and not on the specific values of the rate constants. The deficiency of a reaction network is non-negative because it can be interpreted as the dimension of a certain linear subspace [11] or the codimension of a certain ideal [8]. For systems arising from zero-deficiency networks and networks whose linkage classes have deficiencies zero or one, there are many results due to Feinberg that concern the existence, uniqueness, and stability of steady states [11, 12, 13, 14].

\section{Sufficient CONDITIONS FOR THE EXISTENCE OF TORIC STEAdy STATES}

The main results of this section, Theorems 3.3, 3.8, and 3.19, give sufficient conditions for a chemical reaction system to have toric steady states and state criteria for these systems to have positive toric steady states. Theorems 3.11 and 3.20 give a monomial parametrization of the steady state locus in this case.

We first state several conditions and intermediate results that will lead to Theorem 3.8. Recall that a partition of $\{1,2, \ldots, m\}$ is a collection of nonempty disjoint subsets $I_{1}, I_{2}, \ldots, I_{d}$ with respective cardinalities $l_{1}, l_{2}, \ldots, l_{d}$ such that their union equals $\{1,2, \ldots, m\}$ (or equivalently, such that $\left.l_{1}+l_{2}+\cdots+l_{d}=m\right)$. The $\operatorname{support} \operatorname{supp}(b)$ of a real vector $b \in \mathbb{R}^{m}$ is the subset of indices corresponding to the nonzero entries of $b$. The following condition requires that a certain linear subspace has a basis with disjoint supports.

Condition 3.1. For a chemical reaction system given by a network $G$ with $m$ complexes and reaction rate constants $\kappa_{i j}^{*}$, let $\Sigma$ denote its complex-to-species rate matrix (2.2), and set $d:=$ $\operatorname{dim}(\operatorname{ker}(\Sigma))$. We say that the chemical reaction system satisfies Condition [3.1, if there exists a partition $I_{1}, I_{2}, \ldots, I_{d}$ of $\{1,2, \ldots, m\}$ and a basis $b^{1}, b^{2}, \ldots, b^{d} \in \mathbb{R}^{m}$ of $\operatorname{ker}(\Sigma)$ with $\operatorname{supp}\left(b^{i}\right)=I_{i}$.

Remark 3.2. Conditions 3.1, 3.4, and 3.6 in this article are essentially linear algebra conditions. When we consider a specific choice of rate constants $\kappa_{i j}^{*}$, checking these conditions involves computations over $\mathbb{R}$. However, the objects of interest (such as the subspace in Condition 3.1) are parametrized by the unknown rate constants $\kappa_{i j}$, so verifying the conditions can become quite complicated for large networks. In this case, we need to do linear computations over the field $\mathbb{Q}\left(k_{i j}\right)$ of rational functions on these parameters and check semialgebraic conditions on the rate constants (cf. Remark 3.7).

Condition 3.1 implies that the steady state ideal $J_{\Sigma \Psi}$ is binomial:

Theorem 3.3. Consider a chemical reaction system with $m$ complexes, and let $d$ denote the dimension of $\operatorname{ker}(\Sigma)$. Assume that Condition 3.1 holds (i.e., there exists a partition $I_{1}, I_{2}, \ldots, I_{d}$ of $\{1,2, \ldots, m\}$ and a basis $b^{1}, b^{2}, \ldots, b^{d} \in \mathbb{R}^{m}$ of $\operatorname{ker}(\Sigma)$ with $\left.\operatorname{supp}\left(b^{i}\right)=I_{i}\right)$. Then the steady state ideal $J_{\Sigma \Psi}$ is generated by the binomials

$$
b_{j_{1}}^{j} x^{y_{j_{2}}}-b_{j_{2}}^{j} x^{y_{j_{1}}} \text {, for all } j_{1}, j_{2} \in I_{j} \text {, and for all } 1 \leq j \leq d .
$$

Proof. Consider the vectors $\beta_{j_{1}, j_{2}}^{j}=b_{j_{1}}^{j} e_{j_{2}}-b_{j_{2}}^{j} e_{j_{1}} \in \mathbb{R}^{m}$ for all $j_{1}, j_{2} \in I_{j}$, for all $1 \leq j \leq d$. It is straightforward to check that these vectors span the orthogonal complement $\operatorname{ker}(\Sigma)^{\perp}$ of the kernel of $\Sigma$. But by definition, this complement is spanned by the rows of the matrix $\Sigma$. Therefore, the binomials $b_{j_{1}}^{j} \Psi_{j_{2}}(x)-b_{j_{2}}^{j} \Psi_{j_{1}}(x)$ are $\mathbb{R}$-linear combinations of the polynomials $f_{1}(x), f_{2}(x), \ldots, f_{s}(x)$, and vice-versa. And so the binomials in (3.1) give another system of generators of $J_{\Sigma \Psi}$.

Note that Theorem 3.3 does not provide any information about the existence of (toric) steady states (i.e. real solutions to the binomials (3.1), cf. Definition 2.2), let alone positive steady states. 
In general, this is a question of whether a parametrized family of polynomial systems has real solutions. For this purpose two further conditions are needed:

Condition 3.4. Consider a chemical reaction system given by a network $G$ with $m$ complexes and reaction rate constants $\kappa_{i j}^{*}$ that satisfies Condition 3.1 for the partition $I_{1}, I_{2}, \ldots, I_{d}$ of $\{1,2, \ldots, m\}$ and a basis $b^{1}, b^{2}, \ldots, b^{d} \in \mathbb{R}^{m}$ of $\operatorname{ker}(\Sigma)$ (with $\operatorname{supp}\left(b^{i}\right)=I_{i}$ ). We say that this chemical reaction system additionally satisfies Condition 3.4, if for all $j \in\{1,2, \ldots, d\}$, the nonzero entries of $b^{j}$ have the same sign, that is, if

$$
\operatorname{sign}\left(b_{j_{1}}^{j}\right)=\operatorname{sign}\left(b_{j_{2}}^{j}\right) \text {, for all } j_{1}, j_{2} \in I_{j}, \text { for all } 1 \leq j \leq d .
$$

The next result can be used to check the validity of Condition 3.4

Lemma 3.5. Consider a chemical reaction system with $m$ complexes that satisfies Condition 3.1 for the partition $I_{1}, I_{2}, \ldots, I_{d}$ of $\{1,2, \ldots, m\}$ and the basis $b^{1}, b^{2}, \ldots, b^{d} \in \mathbb{R}^{m}$ of $\operatorname{ker}(\Sigma)$. Let $j \in\{1,2, \ldots, d\}$, There exists an $\left(l_{j}-1\right) \times l_{j}$ submatrix $\Sigma_{j}$ of $\Sigma$ with columns indexed by the elements of $I_{j}$ and linearly independent rows (that is, $\operatorname{rank}\left(\Sigma_{j}\right)=l_{j}-1$ ). Let $\Sigma_{j}$ be any such matrix. For $i \in\left\{1, \ldots, l_{j}\right\}$, call $\Sigma_{j}(i)$ the submatrix of $\Sigma_{j}$ obtained by deleting its $i$-th column. Then the system satisfies Condition 3.4 (that is, equations (3.2) are satisfied) if and only if, for all $j \in\{1,2, \ldots, d\}$, the sign of $\operatorname{det}\left(\Sigma_{j}(i)\right)$ is different from the sign of $\operatorname{det}\left(\Sigma_{j}(i+1)\right)$ for $1 \leq i \leq l_{j}-1$.

Proof. First, note that the kernel of the submatrix of $\Sigma$ formed by the columns indexed by $I_{j}$ has dimension one and is spanned by the vector $b_{j}^{\prime}$ which consists of the $l_{j}$ entries of $b^{j}$ that are indexed by $I_{j}$. So there exist $l_{j}-1$ rows that give a matrix $\Sigma_{j}$ as in the statement.

By a basic result from Linear Algebra, the kernel of $\Sigma_{j}$ is spanned by the vector $v^{\prime}$ with $i$-th entry equal to $(-1)^{i} \operatorname{det}\left(\Sigma_{j}(i)\right)$. As the vector $b_{j}^{\prime}$ must be a multiple of $v^{\prime}$, it is immediate that (3.2) holds if and only if the sign of $\operatorname{det}\left(\Sigma_{j}(i)\right)$ is different from the sign of $\operatorname{det}\left(\Sigma_{j}(i+1)\right)$ for $1 \leq i \leq l_{j}-1$.

Condition 3.4 is necessary for the existence of positive real solutions to the system defined by setting the binomials (3.1) to zero. In working towards sufficiency, observe that the system can be rewritten as

$$
x^{y_{j_{1}}-y_{j_{2}}}=\frac{b_{j_{1}}^{j}}{b_{j_{2}}^{j}}, \text { for all } j_{1}, j_{2} \in I_{j} \text { and for all } 1 \leq j \leq d .
$$

Note that Condition 3.4 implies that the right-hand side of the above equation is positive. In addition, we are interested in positive solutions $x \in \mathbb{R}_{>0}^{s}$, so we now apply $\ln (\cdot)$ to both sides and examine the solvability of the resulting linear system:

$$
\ln x\left(y_{j_{1}}-y_{j_{2}}\right)^{t}=\ln \frac{b_{j_{1}}^{j}}{b_{j_{2}}^{j}} \text {, for all } j_{1}, j_{2} \in I_{j} \text { and for all } 1 \leq j \leq d,
$$

where $\ln x=\left(\ln \left(x_{1}\right), \ln \left(x_{2}\right), \ldots, \ln \left(x_{s}\right)\right)$. Now collect the differences $\left(y_{j_{1}}-y_{j_{2}}\right)^{t}$ as columns of a matrix

$$
\Delta:=\left[\left(y_{j_{1}}-y_{j_{2}}\right)^{t}\right]_{\forall j_{1}, j_{2} \in I_{j}, \forall 1 \leq j \leq d}
$$

and define the (row) vector

$$
\Theta_{\kappa}:=\left(\ln \frac{b_{j_{1}}^{j}}{b_{j_{2}}^{j}}\right)_{\forall j_{1}, j_{2} \in I_{j}, \forall 1 \leq j \leq d} .
$$

Observe that the basis vectors $b^{j}$ and hence the vector $\Theta_{\kappa}$ depend on the rate constants. The binomials (3.1) admit a real positive solution (in the presence of Condition 3.4), if and only if the linear system

$$
(\ln x) \Delta=\Theta_{\kappa}
$$


has a real solution $(\ln x) \in \mathbb{R}^{s}$. This is the motivation for our final condition and Theorem 3.8 below:

Condition 3.6. Consider a chemical reaction system given by a network $G$ with $m$ complexes and reaction rate constants $\kappa_{i j}^{*}$ that satisfies both Condition 3.1 (i.e. there exists a partition $I_{1}, I_{2}, \ldots, I_{d}$ of $\{1,2, \ldots, m\}$ and a basis $b^{1}, b^{2}, \ldots, b^{d} \in \mathbb{R}^{m}$ of $\operatorname{ker}(\Sigma)$ with $\left.\operatorname{supp}\left(b^{i}\right)=I_{i}\right)$ and Condition 3.4 (i.e., the coefficients of each binomial in equation (3.1) are of the same sign). Recall the matrix $\Delta$ and the vector $\Theta_{\kappa}$ (defined in equations (3.3) and (3.4), respectively). Let $U$ be a matrix with integer entries whose columns form a basis of the kernel of $\Delta$, that is, $U$ is an integer matrix of maximum column rank such that the following matrix product is a zero matrix with s rows:

$$
\Delta U=\mathbf{0} .
$$

We say that this chemical reaction system additionally satisfies Condition 3.6 if the linear system (3.5) has a real solution $(\ln x) \in \mathbb{R}^{s}$. Equivalently, the Fundamental Theorem of Linear Algebra [31 implies that equation (3.5) has a solution, if and only if

$$
\Theta_{\kappa} U=0 \text {. }
$$

Remark 3.7. Conditions 3.4 and 3.6 impose semialgebraic constraints on the rate constants:

- If the matrix $\Delta$ defined in (3.3) has full column rank (i.e. the right kernel is trivial), then $U$ is the zero vector. It follows that equation (3.6) holds, and hence, Condition 3.6 is trivially satisfied for any positive vector of rate constants. We will see that this is the case for multisite phosphorylation networks.

- If the matrix $\Delta$ does not have full column rank (i.e. there exists a nontrivial right kernel), then equation (3.6) can be translated to a system of polynomial equations in the rate constants.

Now we can state sufficient conditions for a chemical reaction system to admit positive toric steady states:

Theorem 3.8 (Existence of positive toric steady states). Consider a chemical reaction system with $m$ complexes which satisfies Condition 3.1 and hence has a binomial steady state ideal $J_{\Sigma \Psi}$. Then this chemical reaction system admits a positive toric steady state if and only if Conditions 3.4 and 3.6 hold.

Proof. Assume that Conditions 3.1, 3.4, and 3.6 hold. Lemma 3.5 implies that the coefficients of the binomial system are of the same sign, hence $\Delta$ and $\Theta_{\kappa}$ given in equations (3.3) and (3.4) and the linear system (3.5) are well-defined. Then Condition 3.6 gives a solution $(\ln x)$ to the system (3.5), which immediately gives a positive steady state $x \in \mathbb{R}_{>0}^{s}$ of the chemical reaction system.

On the other hand, assume that Condition 3.1 holds and that the system admits a positive steady state, that is, the binomial system (3.1) has a positive real solution. In this case the coefficients of the binomials must be of the same sign, which implies that Condition 3.4 holds additionally. Again, positive real solutions of the binomial system imply solvability of the linear system (3.5) and thus, Condition 3.6 is satisfied as well.

Remark 3.9 (Existence of steady states using fixed point arguments). In some cases, one can establish the existence of positive steady states by using fixed-point arguments. If the stoichiometric compatibility classes of a network are bounded, a version of the Brouwer fixed point theorem guarantees that a non-negative steady state exists in each compatibility class. If moreover the chemical reaction system has no boundary steady states, we deduce the existence of a positive steady state in each compatibility class. For example, the multisite phosphorylation networks that are studied in this article have this property. The positive conservation laws in (4.1) ensure boundedness and Lemma 4.2 shows that no boundary steady states can occur. 
The focus of our results, however, is slightly different. We are more interested in parametrizing the steady state locus (and hence all positive steady states) and less with the actual number of steady states within a given stoichiometric compatibility class (apart from Section 5, where we are concerned with compatibility classes having at least two distinct positive steady states). Moreover, using fixed point arguments, the existence of positive steady states may only be deduced if the chemical reaction system has no boundary steady states, which is somewhat rare in examples from Computational Biology. Our results do not require any information about boundary steady states.

Example 3.10 (Triangle network, continued). We return to Example 2.3 to illustrate the three conditions. First, $\operatorname{ker}(\Sigma)$ is the plane in $\mathbb{R}^{3}$ orthogonal to the vector $\left(-2 \kappa_{12}-\kappa_{13}, 2 \kappa_{21}+\kappa_{23}, \kappa_{31}-\right.$ $\left.\kappa_{32}\right)$. It follows that the partition $\{1,2\},\{3\}$ works to satisfy Condition 3.1 if and only if $\kappa_{31}=\kappa_{32}$. Therefore, for a chemical reaction system arising from the Triangle network, Condition 3.1 holds (with partition $\{1,2\},\{3\})$ if and only if the system has toric steady states. The forward direction is an application of Theorem 3.3, while for general networks the reverse implication is false: we will see in Example 3.15 that there are networks with toric steady states that do not satisfy Condition 3.1 for any partition.

Next, for those systems for which $\kappa_{31}=\kappa_{32}$, Condition 3.4 comes down to verifying that the entries of the vector $\left(-2 \kappa_{12}-\kappa_{13}, 2 \kappa_{21}+\kappa_{23}\right)$ have opposite signs, which is clearly true for positive rate constants. Finally, Condition 3.6 asks (again, in the $\kappa_{31}=\kappa_{32}$ setting) whether the following linear system has a real solution $\left(\ln x_{1}, \ln x_{2}\right) \in \mathbb{R}^{2}$ :

$$
\left(\ln x_{1}, \ln x_{2}\right) \underbrace{\left(\begin{array}{r}
2 \\
-2
\end{array}\right)}_{=\Delta}=\underbrace{\ln \left(\frac{2 \kappa_{21}+\kappa_{23}}{2 \kappa_{12}+\kappa_{13}}\right)}_{=\Theta_{\kappa}},
$$

which is clearly true. This linear equation arises from the binomial equation

$$
\left(2 \kappa_{12}+\kappa_{13}\right) x_{1}^{2}-\left(2 \kappa_{21}+\kappa_{23}\right) x_{2}^{2}=0 .
$$

As Condition 3.6 holds, Theorem 3.8 implies that these systems admit positive steady states.

Under the hypothesis of Theorem [3.8, the following result shows how to parametrize the steady state locus.

Theorem 3.11. Consider a chemical reaction system that satisfies Conditions 3.1, 3.4, and 3.6. Let $A \in \mathbb{Z}^{w \times s}$ be a matrix of maximal rank $w$ such that $\operatorname{ker}(A)$ equals the span of all the differences $y_{j_{2}}-y_{j_{1}}$ for $j_{1}, j_{2} \in I_{j}$, where $1 \leq j \leq d$. For $1 \leq i \leq s$, we let $A_{i}$ denote the $i$-th column of $A$. Let $\tilde{x} \in \mathbb{R}_{>0}^{s}$ be a positive steady state of the chemical reaction system. Then all positive solutions $x \in \mathbb{R}_{>0}^{s}$ to the binomial system (3.1) can be written as

$$
x=\left(\tilde{x}_{1} t^{A_{1}}, \tilde{x}_{2} t^{A_{2}}, \ldots, \tilde{x}_{s} t^{A_{s}}\right),
$$

for some $t \in \mathbb{R}_{>0}^{w}$ (where we are using the standard notation for multinomial exponents). In particular, the positive steady state locus has dimension $w$ and can be parametrized by monomials in the concentrations. Any two distinct positive steady states $x^{1}$ and $x^{2}$ satisfy

$$
\ln x^{2}-\ln x^{1} \in \operatorname{im}\left(A^{t}\right)=\operatorname{span}\left\{y_{j_{2}}-y_{j_{1}} \mid j_{1}, j_{2} \in I_{j}, 1 \leq j \leq d\right\}^{\perp} .
$$

Proof. By definition, the rows of $A$ span the orthogonal complement of the linear subspace spanned by the differences $y_{j_{2}}-y_{j_{1}}$ for $j_{1}, j_{2} \in I_{j}, 1 \leq j \leq d$. Let $\tilde{x} \in \mathbb{R}_{>0}^{s}$ be a positive steady state of the chemical reaction system; in other words, it is a particular positive solution for the following system of equations:

$$
b_{j_{1}}^{j} x^{y_{j_{2}}}-b_{j_{2}}^{j} x^{y_{j_{1}}}=0 \text { for all } j_{1}, j_{2} \in I_{j} \text {, and for all } 1 \leq j \leq d .
$$

(Here the $b^{j}$ are the basis vectors of $\operatorname{ker}(\Sigma)$ with disjoint support.) Then it follows from basic results on binomial equations that all positive solutions $x \in \mathbb{R}_{>0}^{s}$ to the above system of binomial 
equations can be written as

$$
x=\left(\tilde{x}_{1} t^{A_{1}}, \tilde{x}_{2} t^{A_{2}}, \ldots, \tilde{x}_{s} t^{A_{s}}\right),
$$

for some $t \in \mathbb{R}_{>0}^{w}$. In particular, the positive steady state locus has $w$ degrees of freedom.

For the convenience of the reader, we expand now the previous argument. In fact, it is easy to check that any vector of this shape is a positive solution. We first let $x^{*}$ be a particular positive solution of the above binomials. Then $\frac{x^{*}}{\tilde{x}}:=\left(\frac{x_{1}^{*}}{\tilde{x_{1}}}, \frac{x_{2}^{*}}{\tilde{x_{2}}}, \ldots \frac{x_{s}^{*}}{\tilde{x_{s}}}\right)$ is a positive solution of the system of equations:

$$
x^{y_{j_{2}}}-x^{y_{j_{1}}}=0 \quad \text { for all } j_{1}, j_{2} \in I_{j}, \text { for all } 1 \leq j \leq d .
$$

Therefore, $\left(\frac{x^{*}}{\tilde{x}}\right)^{y_{j_{2}}-y_{j_{1}}}=1$. Or, equivalently, $\ln \left(\frac{x^{*}}{\tilde{x}}\right) \cdot\left(y_{j_{2}}-y_{j_{1}}\right)=0$. This implies that $\ln \left(\frac{x^{*}}{\tilde{x}}\right)$ belongs to the rowspan of $A$, and this means there exist $\lambda_{1}, \lambda_{2}, \ldots, \lambda_{w}$ such that, if $\mathcal{A}_{1}, \mathcal{A}_{2}, \ldots, \mathcal{A}_{w}$ represent the rows of $A$, then we can write

$$
\left(\ln \left(\frac{x^{*}}{\tilde{x}}\right)\right)_{i}=\lambda_{1}\left(\mathcal{A}_{1}\right)_{i}+\lambda_{2}\left(\mathcal{A}_{2}\right)_{i}+\cdots+\lambda_{w}\left(\mathcal{A}_{w}\right)_{i}, \quad \text { for all } 1 \leq i \leq s .
$$

If we call $t_{\ell}:=\exp \left(\lambda_{\ell}\right)$ for $1 \leq \ell \leq w$, then $x_{i}^{*}=\tilde{x}_{i} t^{A_{i}}$ for all $1 \leq i \leq s$, which is what we wanted to prove.

We now turn to the case of a network for which Condition 3.1 holds with the same partition for all choices of rate constants. The following result, which follows immediately from Theorem 3.8 , states that for such a network, the semialgebraic set of rate constants that give rise to systems admitting positive steady states is defined by Conditions 3.4 and 3.6 ,

Corollary 3.12. Let $G$ be a chemical reaction network with $m$ complexes and $r$ reactions, and assume that there exists a partition $I_{1}, I_{2} \ldots, I_{d}$ of the $m$ complexes such that for any choice of reaction rate constants, the resulting chemical reaction system satisfies Condition 3.1 with this partition. Then a vector of reaction rate constants $\kappa_{i j}^{*} \in \mathbb{R}_{>0}^{r}$ gives rise to a system that admits a positive steady state if and only if $\kappa_{i j}^{*}$ satisfies Conditions 3.4 and 3.6 .

In the following example, we see that the 2-site phosphorylation network satisfies the hypothesis of Corollary 3.12. The 2-site system generalizes the 1-site system in Example 2.1, and we will consider general $n$-site systems in Section 4 .

Example 3.13 (2-site phosphorylation system). The dual phosphorylation network arises from the 1-site network (2.4) by allowing a total of two phosphate groups to be added to the substrate of $S_{0}$ rather than only one. Again there are two enzymes ( $E$ and $F)$, but now there are 3 substrates $\left(S_{0}\right.$, $S_{1}$, and $\left.S_{2}\right)$. The substrate $S_{i}$ is the substrate obtained from $S_{0}$ by attaching i phosphate groups to it. Each substrate can accept (via an enzymatic reaction involving $E$ ) or lose (via a reaction involving F) at most one phosphate; this means that the mechanism is "distributive". In addition, we say that the phosphorylation is "sequential" because multiple phosphate groups must be added in a specific order, and removed in a specific order as well.

$$
\begin{aligned}
& S_{0}+E \underset{k_{\text {off }_{0}}}{\stackrel{k_{\text {on }_{0}}}{\rightleftarrows}} E S_{0} \stackrel{k_{\text {cat }}}{\rightarrow} S_{1}+E \underset{k_{\text {off }_{1}}^{\rightleftarrows}}{\stackrel{k_{\text {on }_{1}}}{\rightleftarrows}} E S_{1} \stackrel{k_{\text {cat }_{1}}^{\rightarrow}}{\rightarrow} S_{2}+E \\
& S_{2}+F \underset{l_{\mathrm{off}_{1}}}{\stackrel{l_{\mathrm{on}_{1}}}{\rightleftarrows}} F S_{2} \stackrel{l_{\mathrm{cat}_{1}}}{\rightarrow} S_{1}+F \underset{l_{\mathrm{off}_{0}}}{\stackrel{l_{\mathrm{on}_{0}}}{\rightleftarrows}} F S_{1} \stackrel{l_{\mathrm{cat}_{0}}}{\rightarrow} S_{0}+F
\end{aligned}
$$

We order the 9 species as $\left(S_{0}, S_{1}, S_{2}, E S_{0}, E S_{1}, F S_{1}, F S_{2}, E, F\right)$, and we order the 10 complexes as $\left(S_{0}+E, S_{1}+E, S_{2}+E, E S_{0}, E S_{1}, S_{0}+F, S_{1}+F, S_{2}+F, F S_{1}, F S_{2}\right)$. The $9 \times 10$-matrix $Y^{t}$ and 
the $10 \times 10$-matrix $A_{\kappa}^{t}$ for this system are the following:

$$
\begin{aligned}
& Y^{t}=\left[\begin{array}{llllllllll}
1 & 0 & 0 & 0 & 0 & 1 & 0 & 0 & 0 & 0 \\
0 & 1 & 0 & 0 & 0 & 0 & 1 & 0 & 0 & 0 \\
0 & 0 & 1 & 0 & 0 & 0 & 0 & 1 & 0 & 0 \\
0 & 0 & 0 & 1 & 0 & 0 & 0 & 0 & 0 & 0 \\
0 & 0 & 0 & 0 & 1 & 0 & 0 & 0 & 0 & 0 \\
0 & 0 & 0 & 0 & 0 & 0 & 0 & 0 & 1 & 0 \\
0 & 0 & 0 & 0 & 0 & 0 & 0 & 0 & 0 & 1 \\
1 & 1 & 1 & 0 & 0 & 0 & 0 & 0 & 0 & 0 \\
0 & 0 & 0 & 0 & 0 & 1 & 1 & 1 & 0 & 0
\end{array}\right] \\
& A_{\kappa}^{t}:=\left[\begin{array}{cccccccccc}
-k_{\mathrm{on}_{0}} & 0 & 0 & k_{\mathrm{off}_{0}} & 0 & 0 & 0 & 0 & 0 & 0 \\
0 & -k_{\mathrm{on}_{1}} & 0 & k_{\mathrm{cat}_{0}} & k_{\mathrm{off}_{1}} & 0 & 0 & 0 & 0 & 0 \\
0 & 0 & 0 & 0 & k_{\mathrm{cat}_{1}} & 0 & 0 & 0 & 0 & 0 \\
k_{\mathrm{on}_{0}} & 0 & 0 & -k_{\mathrm{off}_{0}}-k_{\mathrm{cat}_{0}} & 0 & 0 & 0 & 0 & 0 & 0 \\
0 & k_{\mathrm{on}_{1}} & 0 & 0 & -k_{\mathrm{off}_{1}}-k_{\mathrm{cat}_{1}} & 0 & 0 & 0 & 0 & 0 \\
0 & 0 & 0 & 0 & 0 & 0 & 0 & 0 & l_{\mathrm{cat}_{0}} & 0 \\
0 & 0 & 0 & 0 & 0 & 0 & -l_{\mathrm{on}_{0}} & 0 & l_{\mathrm{off}_{0}} & l_{\mathrm{cat}_{1}} \\
0 & 0 & 0 & 0 & 0 & 0 & 0 & -l_{\mathrm{on}_{1}} & 0 & l_{\text {off }_{1}} \\
0 & 0 & 0 & 0 & 0 & 0 & l_{\mathrm{on}_{0}} & 0 & -l_{\mathrm{cat}_{0}}-l_{\text {off }_{0}} & 0 \\
0 & 0 & 0 & 0 & 0 & 0 & 0 & l_{\mathrm{on}_{1}} & 0 & -l_{\mathrm{cat}_{1}}-l_{\mathrm{off}_{1}}
\end{array}\right]
\end{aligned}
$$

We will analyze the steady state locus of the resulting chemical reaction system by focusing on the structure of the kernel of the matrix $\Sigma=Y^{t} A_{\kappa}^{t}$ of the system. Note that the network (3.9) has only two terminal strong linkage classes, $\left\{S_{2}+E\right\}$ and $\left\{S_{0}+F\right\}$. Also, $\operatorname{span}\left\{e_{3}, e_{6}\right\} \subseteq \operatorname{ker}(\Sigma)$, where $e_{i}$ denotes the $i$-th canonical vector of $\mathbb{R}^{10}$. A partition of the 10 complexes that satisfies Condition 3.1 is given by $I_{1}=\{1,4,7,9\}, I_{2}=\{2,5,8,10\}, I_{3}=\{3\}$, and $I_{4}=\{6\}$. A corresponding basis of $\operatorname{ker}(\Sigma)$, that is, one in which the $i$-th basis vector has support $I_{i}$, is:

$$
b^{1}=\left[\begin{array}{c}
\left(k_{\mathrm{off}_{0}}+k_{\mathrm{cat}_{0}}\right) k_{\mathrm{on}_{1}} k_{\mathrm{cat}_{1}} l_{\mathrm{on}_{1}} l_{\mathrm{on}_{0}} l_{\mathrm{cat}_{0}} \\
0 \\
0 \\
k_{\mathrm{on}_{0}} k_{\mathrm{on}_{1}} k_{\mathrm{cat}_{1}} l_{\mathrm{on}_{1}} l_{\mathrm{on}_{0}} l_{\mathrm{cat}_{0}} \\
0 \\
0 \\
k_{\mathrm{on}_{0}} k_{\mathrm{cat}_{0}} k_{\mathrm{on}_{1}} k_{\mathrm{cat}_{1}} l_{\mathrm{on}_{1}}\left(l_{\mathrm{cat}_{0}}+l_{\mathrm{off}_{0}}\right) \\
0 \\
k_{\mathrm{on}_{0}} k_{\mathrm{cat}_{0}} l_{\mathrm{on}_{0}} k_{\mathrm{on}_{1}} k_{\mathrm{cat}_{1}} l_{\mathrm{on}_{1}} \\
0
\end{array}\right], b^{2}=\left[\begin{array}{c}
0 \\
k_{\mathrm{on}_{0}} k_{\mathrm{cat}_{0}} l_{\mathrm{on}_{0}}\left(k_{\mathrm{off}_{1}}+k_{\mathrm{cat}_{1}}\right) l_{\mathrm{on}_{1}} l_{\mathrm{cat}_{1}} \\
0 \\
0 \\
k_{\mathrm{on}_{0}} k_{\mathrm{cat}_{0}} l_{\mathrm{on}_{0}} k_{\mathrm{on}_{1}} l_{\mathrm{on}_{1}} l_{\mathrm{cat}_{1}} \\
0 \\
0 \\
k_{\mathrm{on}_{0}} k_{\mathrm{cat}_{0}} l_{\mathrm{on}_{0}} k_{\mathrm{on}_{1}} k_{\mathrm{cat}_{1}}\left(l_{\mathrm{cat}_{1}}+l_{\mathrm{off}_{1}}\right) \\
0 \\
k_{\mathrm{on}_{0}} k_{\mathrm{cat}_{0}} l_{\mathrm{on}_{0}} k_{\mathrm{on}_{1}} k_{\mathrm{cat}_{1}} l_{\mathrm{on}_{1}}
\end{array}\right], b^{3}=e_{3}, b^{4}=e_{6} .
$$

The structure of this basis $\left\{b^{i}\right\}$ implies that for $v \in \mathbb{R}^{10}, v \in \operatorname{ker}(\Sigma)$ if and only if $v$ satisfies the following binomial equations:

$$
\begin{aligned}
& b_{1}^{1} v_{4}-b_{4}^{1} v_{1}=0, \quad b_{2}^{2} v_{5}-b_{5}^{2} v_{2}=0, \\
& b_{1}^{1} v_{7}-b_{7}^{1} v_{1}=0, \quad b_{2}^{2} v_{8}-b_{8}^{2} v_{2}=0, \\
& b_{1}^{1} v_{9}-b_{9}^{1} v_{1}=0, \quad b_{2}^{2} v_{10}-b_{10}^{2} v_{2}=0,
\end{aligned}
$$

Hence, any steady state of the 2-site phosphorylation system must satisfy the following equations in the species concentrations $x=\left(x_{S_{0}}, x_{S_{1}}, \ldots, x_{E}, x_{F}\right)$ :

$$
\begin{array}{ll}
b_{1}^{1} x_{4}-b_{4}^{1} x_{8} x_{1}=0, & b_{2}^{2} x_{5}-b_{5}^{2} x_{8} x_{2}=0, \\
b_{1}^{1} x_{9} x_{2}-b_{7}^{1} x_{8} x_{1}=0, & b_{2}^{2} x_{9} x_{3}-b_{8}^{2} x_{8} x_{2}=0, \\
b_{1}^{1} x_{6}-b_{9}^{1} x_{8} x_{1}=0, & b_{2}^{2} x_{7}-b_{10}^{2} x_{8} x_{2}=0 .
\end{array}
$$

To check Condition 3.6, we consider the matrix $\Delta$ and the vector $\Theta_{\kappa}$ :

$\Delta=\left[e_{4}-e_{8}-e_{1}\left|e_{9}+e_{2}-e_{8}-e_{1}\right| e_{6}-e_{8}-e_{1}\left|e_{5}-e_{8}-e_{2}\right| e_{9}+e_{3}-e_{8}-e_{2} \mid e_{7}-e_{8}-e_{2}\right]$ 


$$
\Theta_{\kappa}=\left(\ln \frac{b_{4}^{1}}{b_{1}^{1}}, \ln \frac{b_{7}^{1}}{b_{1}^{1}}, \ln \frac{b_{9}^{1}}{b_{1}^{1}}, \ln \frac{b_{5}^{2}}{b_{2}^{2}}, \ln \frac{b_{8}^{2}}{b_{2}^{2}}, \ln \frac{b_{10}^{2}}{b_{2}^{2}}\right) .
$$

It is straightforward to check that $\Delta$ has rank 6 and hence full rank. Thus Condition 3.6 is trivially satisfied and does not pose any constraints on the rate constants.

Following the proof of Theorem 3.11, we first will parametrize the solution set of the following reduced system:

$$
\begin{array}{ll}
x_{4}-x_{8} x_{1}=0, & x_{5}-x_{8} x_{2}=0, \\
x_{9} x_{2}-x_{8} x_{1}=0, & x_{9} x_{3}-x_{8} x_{2}=0, \\
x_{6}-x_{8} x_{1}=0, & x_{7}-x_{8} x_{2}=0 .
\end{array}
$$

We are interested in an integer matrix $A$ such that $\operatorname{ker}(A)=\operatorname{im}(\Delta)$. One such matrix is

$$
A=\left(\begin{array}{lllllllll}
0 & 1 & 2 & 1 & 2 & 1 & 2 & 1 & 0 \\
0 & 0 & 0 & 1 & 1 & 1 & 1 & 1 & 1 \\
1 & 1 & 1 & 1 & 1 & 1 & 1 & 0 & 0
\end{array}\right)
$$

This provides the following 3-dimensional parametrization of the reduced system:

$$
\left(t_{1}, t_{2}, t_{3}\right) \mapsto\left(t_{3}, t_{1} t_{3}, t_{1}^{2} t_{3}, t_{1} t_{2} t_{3}, t_{1}^{2} t_{2} t_{3}, t_{1} t_{2} t_{3}, t_{1}^{2} t_{2} t_{3}, t_{1} t_{2}, t_{2}\right),
$$

where $t_{2}$ is the concentration of the enzyme $F, t_{1}$ is the quotient of the concentration of the enzyme $E$ divided by the concentration of the enzyme $F$, and $t_{3}$ is the concentration of the substrate $S_{0}$. Returning to the original binomials (3.11), we have the following particular solution:

$$
x_{1}^{*}=x_{8}^{*}=x_{9}^{*}=1, x_{2}^{*}=\frac{b_{7}^{1}}{b_{1}^{1}}, x_{3}^{*}=\frac{b_{8}^{2} b_{7}^{1}}{b_{1}^{1} b_{2}^{2}}, x_{4}^{*}=\frac{b_{4}^{1}}{b_{1}^{1}}, x_{5}^{*}=\frac{b_{5}^{2} b_{7}^{1}}{b_{1}^{1} b_{2}^{2}}, x_{6}^{*}=\frac{b_{9}^{1}}{b_{1}^{1}}, x_{7}^{*}=\frac{b_{10}^{2} b_{7}^{1}}{b_{1}^{1} b_{2}^{2}} .
$$

Therefore we obtain the following 3-dimensional parametrization of the positive steady state locus of (3.11), as predicted in Theorem 3.11:

$$
\begin{aligned}
\mathbb{R}_{>0}^{3} & \rightarrow \mathbb{R}_{>0}^{9} \\
\left(t_{1}, t_{2}, t_{3}\right) & \mapsto\left(t_{3}, \frac{b_{7}^{1}}{b_{1}^{1}} t_{1} t_{3}, \frac{b_{8}^{2} b_{7}^{1}}{b_{1}^{1} b_{2}^{2}} t_{1}^{2} t_{3}, \frac{b_{4}^{1}}{b_{1}^{1}} t_{1} t_{2} t_{3}, \frac{b_{5}^{2} b_{7}^{1}}{b_{1}^{1} b_{2}^{2}} t_{1}^{2} t_{2} t_{3}, \frac{b_{9}^{1}}{b_{1}^{1}} t_{1} t_{2} t_{3}, \frac{b_{10}^{2} b_{7}^{1}}{b_{1}^{1} b_{2}^{2}} t_{1}^{2} t_{2} t_{3}, t_{1} t_{2}, t_{2}\right) .
\end{aligned}
$$

Recall that the values $b_{j}^{i}$ are polynomials in the rate constants shown in the display of the vectors $b^{1}$ and $b^{2}$. Finally, note that none of the calculations in this example depends on the specific values of the rate constants; in particular, one partition works for all systems, so the hypothesis of Corollary 3.12 holds.

3.1. More general sufficient conditions. We show in Example 3.15 below, extracted from [30], that the conditions in Theorem 3.8 are not necessary for a chemical reaction system to have toric steady states; in other words, the converse of Theorem 3.8 does not hold. However, the condition for the steady state ideal to be generated by binomials always can be checked algorithmically via a Gröbner basis computation, as stated in the following lemma.

Lemma 3.14 (Proposition 1.1.(a) of [10]). Let I be a binomial ideal, let $\prec$ be a monomial order, and let $G$ be the reduced Gröbner basis of I for that ordering. Then $G$ consists of binomials.

Lemma 3.14 is a basic result about binomial ideals which is due to Eisenbud and Sturmfels [10]; it is a result concerning polynomial linear combinations. Note however that Theorem 3.8 requires only linear algebra computations over $\mathbb{R}$. We make use of Lemma 3.14 in the following example. We will return to it later to show that Theorem 3.19 below can be used to prove that this system has toric steady states, without needing to compute a Gröbner basis. 
Example 3.15 (Shinar and Feinberg network). This example demonstrates that Condition [3.1] is not necessary for a chemical reaction system to have toric steady states. The network in Example (S60) of the Supporting Online Material of the recent article of Shinar and Feinberg is the following [30]:

$$
\begin{array}{r}
X D \underset{\kappa_{21}}{\stackrel{\kappa_{12}}{\rightleftarrows}} X \underset{\kappa_{32}}{\stackrel{\kappa_{23}}{\rightleftarrows}} X T \stackrel{\kappa_{34}}{\rightarrow} X_{p} \\
X_{p}+Y \underset{\kappa_{65}}{\stackrel{\kappa_{56}}{\rightleftarrows}} X_{p} Y \stackrel{\kappa_{67}}{\rightarrow} X+Y_{p} \\
X T+Y_{p} \underset{\kappa_{898}}{\stackrel{\kappa_{89}}{\rightleftarrows}} X T Y_{p} \stackrel{\kappa_{9,10}}{\longrightarrow} X T+Y \\
X D+Y_{p} \underset{\kappa_{12,11}}{\stackrel{\kappa_{11,12}}{\rightleftarrows}} X D Y_{p} \stackrel{\kappa_{12,13}}{\rightarrow} X D+Y
\end{array}
$$

We denote by $x_{1}, x_{2}, \ldots, x_{9}$ the concentrations of the species as follows:

$$
\begin{gathered}
x_{X D}=x_{1}, x_{X}=x_{2}, x_{X T}=x_{3}, x_{X_{p}}=x_{4} \\
x_{Y}=x_{5}, x_{X_{p} Y}=x_{6}, x_{Y_{p}}=x_{7}, x_{X T Y_{p}}=x_{8}, x_{X D Y_{p}}=x_{9} .
\end{gathered}
$$

Note that the numbering of the 13 complexes in the network is reflected in the names of the rate constants $\kappa_{i j}$. The chemical reaction system is the following:

$$
\begin{aligned}
\frac{d x_{1}}{d t} & =-\kappa_{12} x_{1}+\kappa_{21} x_{2}-\kappa_{11,12} x_{1} x_{7}+\left(\kappa_{12,11}+\kappa_{12,13}\right) x_{9} \\
\frac{d x_{2}}{d t} & =\kappa_{12} x_{1}+\left(-\kappa_{21}-\kappa_{23}\right) x_{2}+\kappa_{32} x_{3}+\kappa_{67} x_{6} \\
\frac{d x_{3}}{d t} & =\kappa_{23} x_{2}+\left(-\kappa_{32}-\kappa_{34}\right) x_{3}-\kappa_{89} x_{3} x_{7}+\left(\kappa_{98}+\kappa_{9,10}\right) x_{8} \\
\frac{d x_{4}}{d t} & =\kappa_{34} x_{3}-\kappa_{56} x_{4} x_{5}+\kappa_{65} x_{6} \\
\frac{d x_{5}}{d t} & =-\kappa_{56} x_{4} x_{5}+\kappa_{65} x_{6}+\kappa_{9,10} x_{8}+\kappa_{12,13} x_{9} \\
\frac{d x_{6}}{d t} & =\kappa_{56} x_{4} x_{5}+\left(-\kappa_{65}-\kappa_{67}\right) x_{6} \\
\frac{d x_{7}}{d t} & =\kappa_{67} x_{6}-\kappa_{89} x_{3} x_{7}+\kappa_{98} x_{8}-\kappa_{11,12} x_{1} x_{7}+\kappa_{12,11} x_{9} \\
\frac{d x_{8}}{d t} & =\kappa_{89} x_{3} x_{7}+\left(-\kappa_{98}-\kappa_{9,10}\right) x_{8} \\
\frac{d x_{9}}{d t} & =\kappa_{11,12} x_{1} x_{7}+\left(-\kappa_{12,11}-\kappa_{12,13}\right) x_{9}
\end{aligned}
$$

The reduced Gröbner basis with respect to the lexicographical order $x_{1}>x_{2}>x_{4}>x_{5}>x_{6}>x_{8}>$ $x_{9}>x_{3}>x_{7}$ consists of the following binomials:

$$
\begin{aligned}
g_{1}= & {\left[\kappa_{89} \kappa_{12} \kappa_{23} \kappa_{9,10}\left(\kappa_{12,11}+\kappa_{12,13}\right)+\kappa_{11,12} \kappa_{21} \kappa_{12,13}\left(\kappa_{98}+\kappa_{9,10}\right)\left(\kappa_{32}+\kappa_{34}\right)\right] x_{3} x_{7}+} \\
& +\left[-\kappa_{23} \kappa_{34} \kappa_{12}\left(\kappa_{12,11}+\kappa_{12,13}\right)\left(\kappa_{98}+\kappa_{9,10}\right)\right] x_{3} \\
g_{2}= & {\left[-\kappa_{11,12} \kappa_{21} \kappa_{34}\left(\kappa_{98}+\kappa_{9,10}\right)\left(\kappa_{32}+\kappa_{34}\right)\right] x_{3}+} \\
& +\left[\kappa_{11,12} \kappa_{21} \kappa_{12,13}\left(\kappa_{98}+\kappa_{9,10}\right)\left(\kappa_{32}+\kappa_{34}\right)+\kappa_{12} \kappa_{23} \kappa_{89} \kappa_{9,10}\left(\kappa_{12,11}+\kappa_{12,13}\right)\right] x_{9} \\
g_{3}= & {\left[-\kappa_{23} \kappa_{34} \kappa_{89} \kappa_{12}\left(\kappa_{12,11}+\kappa_{12,13}\right)\right] x_{3}+} \\
& \quad+\left[\kappa_{23} \kappa_{9,10} \kappa_{89} \kappa_{12}\left(\kappa_{12,11}+\kappa_{12,13}\right)+\kappa_{11,12} \kappa_{21} \kappa_{12,13}\left(\kappa_{98}+\kappa_{9,10}\right)\left(\kappa_{32}+\kappa_{34}\right)\right] x_{8} \\
g_{4}= & \kappa_{67} x_{6}-\kappa_{34} x_{3} \\
g_{5}= & \kappa_{56} \kappa_{67} x_{4} x_{5}+\kappa_{34}\left(-\kappa_{65}-\kappa_{67}\right) x_{3} \\
g_{6}= & \kappa_{23} x_{2}+\left(-\kappa_{32}-\kappa_{34}\right) x_{3} \\
g_{7}= & -\kappa_{21}\left(\kappa_{32}+\kappa_{34}\right) x_{3}+\kappa_{12} \kappa_{23} x_{1}
\end{aligned}
$$

Therefore, the network has toric steady states (for any choice of positive reaction rate constants) because the steady state ideal can be generated by $g_{1}, g_{2}, \ldots, g_{7}$. However, we claim that this chemical reaction system does not satisfy Condition [3.1. In fact, for any rate constants, it is not possible to find a partition $I_{1}, I_{2}, \ldots, I_{6} \subseteq\{1,2, \ldots, 13\}$ such that $\operatorname{ker}(\Sigma)$ has a basis $\left\{b^{1}, b^{2}, \ldots, b^{6}\right\}$ 
with $\operatorname{supp}\left(b^{i}\right)=I_{i}$. This can be seen by noting that the kernel of $\Sigma$ can be generated as follows:

$$
\begin{aligned}
\operatorname{ker}(\Sigma)=\left\langle e_{4},\right. & e_{7}, e_{10}, e_{13},\left(\frac{\kappa_{21} \kappa_{12,13}\left(\kappa_{32}+\kappa_{34}\right)}{\kappa_{23} \kappa_{34} \kappa_{12}}\right) e_{1}+\left(\frac{\kappa_{12,13}\left(\kappa_{32}+\kappa_{34}\right)}{\kappa_{23} \kappa_{34}}\right) e_{2}+\left(\frac{\kappa_{12,13}}{\kappa_{34}}\right) e_{3}+ \\
& +\left(\frac{\left(\kappa_{65}+\kappa_{67}\right) \kappa_{12,13}}{\kappa_{67} \kappa_{56}}\right) e_{5}+\left(\frac{\kappa_{12,13}}{\kappa_{67}}\right) e_{6}+\left(\frac{\left(\kappa_{12,11}+\kappa_{12,13}\right)}{\kappa_{11,12}}\right) e_{11}+e_{12}, \\
& \left(\frac{\kappa_{21} \kappa_{9,10}\left(\kappa_{32}+\kappa_{34}\right)}{\kappa_{23} \kappa_{34} \kappa_{12}}\right) e_{1}+\left(\frac{\kappa_{9,10}\left(\kappa_{32}+\kappa_{34}\right)}{\kappa_{23} \kappa_{34}}\right) e_{2}+\left(\frac{\kappa_{9,10}}{\kappa_{34}}\right) e_{3}+ \\
& \left.+\left(\frac{\left(\kappa_{65}+\kappa_{67}\right) \kappa_{9,10}}{\kappa_{67} \kappa_{56}}\right) e_{5}+\left(\frac{\kappa_{9,10}}{\kappa_{67}}\right) e_{6}+\left(\frac{\kappa_{98}+\kappa_{9,10}}{\kappa_{89}}\right) e_{8}+e_{9}\right\rangle .
\end{aligned}
$$

Our next result, Theorem 3.19, will generalize Theorem 3.8 by giving a stronger condition that guarantees that the steady state locus is generated by binomials. We first need to generalize Conditions 3.1, 3.4, and 3.6 to any (finite) polynomial system.

First we must introduce some notation. For polynomials $F_{1}, F_{2}, \ldots, F_{s^{\prime}} \in \mathbb{R}\left[x_{1}, x_{2}, \ldots, x_{s}\right]$, we denote by $x^{y_{1}}, x^{y_{2}}, \ldots, x^{y_{m^{\prime}}}$ the monomials that occur in these polynomials; that is, there exist $F_{i j} \in \mathbb{R}$ such that $F_{i}(x)=\sum_{j=1}^{m^{\prime}} F_{i j} x^{y_{j}}$ for $i=1,2, \ldots, s^{\prime}$. We can write the polynomial system $F_{1}(x)=F_{2}(x)=\cdots=F_{s^{\prime}}(x)=0$ as

$$
\Sigma^{\prime} \cdot \Psi^{\prime}(x)=0,
$$

where $\Sigma^{\prime}=\left(F_{i j}\right) \in \mathbb{R}^{s^{\prime} \times m^{\prime}}$ is the coefficient matrix and $\Psi^{\prime}(x)=\left(x^{y_{1}}, x^{y_{2}}, \ldots, x^{y_{m^{\prime}}}\right)^{t}$. We will let $d^{\prime}$ denote the dimension of $\operatorname{ker}\left(\Sigma^{\prime}\right)$.

Condition 3.16. We say that the polynomial system (3.18) satisfies Condition 3.16 if there exists a partition $I_{1}, I_{2}, \ldots, I_{d^{\prime}}$ of $\left\{1,2, \ldots, m^{\prime}\right\}$ and a basis $b^{1}, b^{2}, \ldots, b^{d^{\prime}} \in \mathbb{R}^{m^{\prime}}$ of $\operatorname{ker}\left(\Sigma^{\prime}\right)$ such that $\operatorname{supp}\left(b^{i}\right)=I_{i}$.

Condition 3.17. Consider a polynomial system (3.18) that satisfies Condition 3.16 for the partition $I_{1}, I_{2}, \ldots, I_{d^{\prime}}$ of $\left\{1,2, \ldots, m^{\prime}\right\}$ and a basis $b^{1}, b^{2}, \ldots, b^{d^{\prime}} \in \mathbb{R}^{m^{\prime}}$ of $\operatorname{ker}\left(\Sigma^{\prime}\right)$ (with $\left.\operatorname{supp}\left(b^{i}\right)=I_{i}\right)$. We say that the system satisfies additionally Condition 3.17 , if for all $j \in\left\{1,2, \ldots, d^{\prime}\right\}$, the nonzero entries of $b^{j}$ have the same sign.

As before, we collect the differences of exponent vectors as columns of a matrix

$$
\Delta^{\prime}:=\left[\left(y_{j_{1}}-y_{j_{2}}\right)^{t}\right]_{\forall j_{1}, j_{2} \in I_{j}, \forall 1 \leq j \leq d^{\prime}}
$$

and define the (row) vector

$$
\Theta^{\prime}:=\left(\ln \frac{b_{j_{2}}^{j}}{b_{j_{1}}^{j}}\right)_{\forall j_{1}, j_{2} \in I_{j}, \forall 1 \leq j \leq d^{\prime}} .
$$

Condition 3.18. Consider a polynomial system (3.18) which satisfies Conditions [3.16] and 3.17. Let $U^{\prime}$ be a matrix with integer entries whose columns form a basis of the kernel of $\Delta^{\prime}$. We say that this system satisfies additionally Condition 3.18, if the following holds:

$$
\Theta^{\prime} U^{\prime}=0 \text {. }
$$

We then have the following sufficient conditions:

Theorem 3.19. Consider a chemical reaction system with $m$ complexes and assume that there exist monomials $\mathbf{x}^{\alpha_{1}}, \mathbf{x}^{\alpha_{2}}, \ldots, \mathbf{x}^{\alpha_{\ell}}$ and indices $i_{1}, i_{2}, \ldots, i_{\ell}$, with $\left\{i_{1}, i_{2}, \ldots, i_{\ell}\right\} \subseteq\{1,2, \ldots, s\}$, such that Condition 3.16 holds for the enlarged polynomial system

$$
f_{1}=\cdots=f_{s}=\mathbf{x}^{\alpha_{1}} f_{i_{1}}=\cdots=\mathbf{x}^{\alpha_{\ell}} f_{i_{\ell}}=0 .
$$

Then the steady state ideal $J_{\Sigma \psi}$ is binomial.

Moreover, the system has positive (toric) steady states if and only if Conditions 3.17 and 3.18 hold additionally for the enlarged system. 
This theorem can be proved following the lines of the proof of Theorem 3.8 for the enlarged system defined in the statement. It is important to note that the ideal $\left\langle f_{1}, f_{2}, \ldots, f_{s}\right\rangle$ equals the ideal $\left\langle f_{1}, \ldots, f_{s}, \mathbf{x}^{\alpha_{1}} f_{i_{1}}, \ldots, \mathbf{x}^{\alpha_{\ell}} f_{i_{\ell}}\right\rangle$.

With similar proof as in Theorem 3.11, we moreover have:

Theorem 3.20. Under the hypotheses of Theorem 3.19, the steady state locus can be parametrized by monomials in the concentrations.

Remark 3.21. As with Conditions 3.1, 3.4, and 3.6, checking the Conditions in the statement of Theorem 3.19 involves linear algebra computations over $\mathbb{R}$ for fixed rate constants or over $\mathbb{Q}\left(k_{i j}\right)$ for generic parameters, once the monomials $x^{\alpha_{i}}$ are given. In small cases, such monomials can be guessed. In the following example, they were traced in the standard algorithm for the computation of a Gröbner basis of the ideal $\left\langle f_{1}, \ldots, f_{s}\right\rangle$.

We end this section by returning to Example 3.15 .

Example 3.22 (Shinar and Feinberg network, continued). Consider the system of equations:

$$
\left\{\begin{array}{l}
f_{1}=0 \\
f_{2}=0 \\
\vdots \\
f_{9}=0 \\
x_{7} f_{1}=0 \\
x_{7} f_{3}=0 \\
x_{7} f_{8}=0 \\
x_{7} f_{9}=0
\end{array},\right.
$$

This enlarged system satisfies Conditions 3.16 and 3.17 for the following partition:

$$
I_{1}=\{4\}, I_{2}=\{10\}, I_{3}=\{13\}, I_{4}=\{14,15\}, I_{5}=\{16,17\}, I_{6}=\{1,2,3,5,6,7,8,9,11,12\}
$$

and the following basis $b^{1}, b^{2}, \ldots, b^{6}$ of its kernel verifying $\operatorname{supp}\left(b^{j}\right)=I_{j}$ :

$$
\begin{aligned}
& b^{1}=e_{4}, \quad b^{2}=e_{10}, \quad b^{3}=e_{13}, \quad b^{4}=\left(k_{12,11}+k_{12,13}\right) e_{14}+k_{11,12} e_{15}, \quad b^{5}=\left(k_{98}+k_{910}\right) e_{16}+k_{89} e_{17}, \\
& b^{6}=\left(k_{12} k_{23} k_{89} k_{9,10}\left(k_{12,11}+k_{12,13}\right)+k_{21} k_{11,12} k_{12,13}\left(k_{32}+k_{34}\right)\left(k_{98}+k_{9,10}\right)\right) k_{21}\left(k_{32}+k_{34}\right) k_{56} k_{67} e_{1}+ \\
&\left(k_{12} k_{23} k_{89} k_{9,10}\left(k_{12,11}+k_{12,13}\right)+k_{21} k_{11,12} k_{12,13}\left(k_{32}+k_{34}\right)\left(k_{98}+k_{9,10}\right)\right) k_{12}\left(k_{32}+k_{34}\right) k_{56} k_{67} e_{2}+ \\
&\left(k_{12} k_{23} k_{89} k_{9,10}\left(k_{12,11}+k_{12,13}\right)+k_{21} k_{11,12} k_{12,13}\left(k_{32}+k_{34}\right)\left(k_{98}+k_{9,10}\right)\right) k_{12} k_{23} k_{56} k_{67} e_{3}+ \\
&\left(k_{12} k_{23} k_{89} k_{9,10}\left(k_{12,11}+k_{12,13}\right)+k_{21} k_{11,12} k_{12,13}\left(k_{32}+k_{34}\right)\left(k_{98}+k_{9,10}\right)\right) k_{12} k_{23} k_{34}\left(k_{65}+k_{67}\right) e_{5}+ \\
&\left(k_{12} k_{23} k_{89} k_{9,10}\left(k_{12,11}+k_{12,13}\right)+k_{21} k_{11,12} k_{12,13}\left(k_{32}+k_{34}\right)\left(k_{98}+k_{9,10}\right)\right) k_{12} k_{23} k_{34} k_{56} e_{6}+ \\
& k_{12}^{2} k_{23} k_{34}\left(k_{32}+k_{34}\right) k_{56} k_{67}\left(k_{98}+k_{9,10}\right)\left(k_{12,11}+k_{12,13}\right) e_{7}+ \\
& k_{12}^{2} k_{23}^{2} k_{34} k_{56} k_{67}\left(k_{98}+k_{9,10}\right)\left(k_{12,11}+k_{12,13}\right) e_{8}+k_{12}^{2} k_{23}^{2} k_{34} k_{56} k_{67} k_{89}\left(k_{12,11}+k_{12,13}\right) e_{9}+ \\
& k_{12} k_{21} k_{23} k_{34}\left(k_{32}+k_{34}\right) k_{56} k_{67}\left(k_{98}+k_{9,10}\right)\left(k_{12,11}+k_{12,13}\right) e_{11}+ \\
& k_{12} k_{21} k_{23} k_{34}\left(k_{32}+k_{34}\right) k_{56} k_{67}\left(k_{98}+k_{9,10}\right) k_{11,12} e_{12} .
\end{aligned}
$$

In addition to the monomials already occurring in $f_{1}, f_{2}, \ldots, f_{9}$, the following 4 monomials are also in the augmented system: $x^{y_{14}}=x_{1} x_{7}^{2}, x^{y_{15}}=x_{9} x_{7}, x^{y_{16}}=x_{3} x_{7}^{2}$, and $x^{y_{17}}=x_{8} x_{7}$. By Theorem 3.19, the system has toric steady states. Recall that the binomials $g_{1}, g_{2}, \ldots, g_{7}$ in equation (3.16) generate the ideal $\left\langle f_{1}, f_{2}, \ldots, f_{9}\right\rangle=\left\langle f_{1}, f_{2}, \ldots, f_{9}, x_{7} f_{1}, x_{7} f_{3}, x_{7} f_{8}, x_{7} f_{9}\right\rangle$. We can see immediately that there are positive steady states for any choice of positive rate constants, and so there is no need to check Condition 3.18 . 


\section{The $n$-Site PhOSPhORYlation System has toriC STEAdy STATES}

In this section we introduce the $n$-site phosphorylation system (under the assumption of a distributive and sequential mechanism). To show that these systems have toric steady states, we apply Theorem [3.8, this generalizes Example 3.13 (the $n=2$ case). Further, we note that the parametrization of the steady state locus given by Theorem 3.11 is implicit in work of Wang and Sontag [36].

4.1. The $n$-site phosphorylation system. We now define the $n$-site phosphorylation system (also called a "multiple futile cycle") $\Sigma_{n}(\kappa, \mathcal{C})$, which depends on a choice of rate constants $\kappa \in \mathbb{R}_{>0}^{6 n}$ and values of the conservation relations $\mathcal{C}=\left(E_{\text {tot }}, F_{\text {tot }}, S_{\text {tot }}\right) \in \mathbb{R}_{>0}^{3}$. As in the earlier example of the 1-site network (2.4) and the 2-site network (3.9), we will make the assumption of a "distributive" and "sequential" mechanism (see, for example, [6]). As discussed in the Introduction, this $n$-site phosphorylation system is of great biochemical importance: it is a recurring network motif in many networks describing processes as diverse as intracellular signaling (e.g. MAPK signaling with $n=2$ and $n=3$ ), cell cycle control (e.g. Sic1 with $n=9$ ), and cellular differentiation (e.g. NFAT with $n=13)$.

Following notation of Wang and Sontag [36], the $n$-site phosphorylation system arises from the following reaction network:

$$
\begin{aligned}
& S_{0}+E \underset{k_{\mathrm{Off}_{0}}}{\stackrel{k_{\mathrm{On}_{0}}}{\leftrightarrows}} E S_{0} \stackrel{k}{\mathrm{cat}_{0}} S_{1}+E \\
& S_{1}+F \underset{l_{\mathrm{off}_{0}}}{\stackrel{l_{\mathrm{on}_{0}}}{\rightleftarrows}} F S_{1} \stackrel{l^{\mathrm{cat}_{0}}}{\rightarrow} S_{0}+F \\
& \vdots \\
& S_{n-1}+E \underset{k_{\mathrm{Off}_{n-1}}}{\stackrel{k_{\mathrm{On}_{n-1}}}{\rightleftarrows}} E S_{n-1} \stackrel{{ }^{k} \mathrm{cat}_{n-1}}{\rightarrow} S_{n}+E \\
& S_{n}+F \underset{\text { off }_{n-1}}{\stackrel{l_{\mathrm{On}_{n-1}}}{\rightleftarrows}} F S_{n} \stackrel{{ }^{\mathrm{cat}_{n-1}}}{\rightarrow} S_{n-1}+F
\end{aligned}
$$

We see that the $n$-site network has $3 n+3$ chemical species $S_{0}, \ldots, S_{n}, E S_{0}, \ldots, E S_{n-1}, F S_{1}, \ldots, F S_{n}$, $E$, and $F$, so we write a concentration vector as $x=\left(s_{0}, \ldots, s_{n}, c_{0}, \ldots, c_{n-1}, d_{1}, \ldots, d_{n}, e, f\right)$, which is a positive vector of length $3 n+3$. These species comprise $4 n+2$ complexes, and there are $6 n$ reactions. Each reaction has a reaction rate, and we collect these in the vector of rate constants $\kappa=\left(k_{\mathrm{on}_{0}}, \ldots, l_{\mathrm{cat}_{\mathrm{n}-1}}\right) \in \mathbb{R}_{>0}^{6 n}$.

For our purposes, we will introduce the following numbering for the complexes (which is compatible with the numbering in Examples 2.1 and 3.13):

$$
\begin{aligned}
& 1 \rightleftarrows n+2 \rightarrow 2 \\
& 2 \rightleftarrows n+3 \rightarrow 3 \\
& \vdots \\
& n \rightleftarrows 2 n+1 \rightarrow n+1
\end{aligned}
$$

$$
\begin{aligned}
2 n+3 & \rightleftarrows 3 n+3 \rightarrow 2 n+2 \\
2 n+4 & \rightleftarrows 3 n+4 \rightarrow 2 n+3 \\
\vdots & \\
3 n+2 & \rightleftarrows 4 n+2 \rightarrow 3 n+1
\end{aligned}
$$

The conservation relations here correspond to the fact that the total amounts of free and bound enzyme or substrate remain constant. That is, the following three conservation values 
$\mathcal{C}=\left(E_{\text {tot }}, F_{\text {tot }}, S_{\text {tot }}\right) \in \mathbb{R}_{>0}^{3}$ remain unchanged as the dynamical system progresses:

$$
\begin{aligned}
& E_{\mathrm{tot}}=e+\sum_{i=0}^{n-1} c_{i}, \\
& F_{\mathrm{tot}}=f+\sum_{i=1}^{n} d_{i}, \\
& S_{\mathrm{tot}}=\sum_{i=0}^{n} s_{i}+\sum_{i=0}^{n-1} c_{i}+\sum_{i=1}^{n} d_{i} .
\end{aligned}
$$

Any choice of these three values defines a bounded stoichiometric compatibility class of dimension $3 n$ :

$$
\mathcal{P}_{\mathcal{C}}=\left\{x \in \mathbb{R}_{\geq 0}^{3 n+3} \mid \text { the conservation equations (4.1) hold }\right\}
$$

Note that the right hand side of each of the three conservation relations (4.1) is defined by a vector $z_{i} \in S^{\perp}, i=1,2,3$. These vectors play an important role in the following remark and in Lemma4.2 below.

Remark 4.1 (Positive steady states by fixed point arguments). As indicated in Remark 3.9 one may deduce the existence of at least one positive steady state in each stoichiometric compatibility class $\mathcal{P}_{\mathcal{C}}$ (defined by positive $\mathcal{C}$ ) by fixed-point arguments, provided (i) $\mathcal{P}_{\mathcal{C}}$ is bounded and (ii) there are no boundary steady states in any stoichiometric compatibility class $\mathcal{P}_{\mathcal{C}}$ (with $\mathcal{C} \in \mathbb{R}_{>0}^{3}$ ). Point (i) follows from the definition of $\mathcal{P}_{\mathcal{C}} \in \mathbb{R}_{>0}^{3}$ given above. With respect to (ii), we point to Lemma 4.2 below (which can be established by a straightforward generalization of the analysis due to Angeli, De Leenheer, and Sontag in Examples 1 and 2 in [1, $\S 6]$ ).

Lemma 4.2. Let $x^{*} \in \mathbb{R}_{>0}^{s}-\mathbb{R}_{>0}^{s}$ be a boundary steady state. Set $\Lambda:=\left\{i \in\{1, \ldots, s\}: x_{i}^{*}=0\right\}$. Then, $\Lambda$ contains the support of at least one of the vectors $z_{i} \in S^{\perp}$ defining the conservation relations (4.1). Therefore, there are no boundary steady states in any stoichiometric compatibility class $\mathcal{P}_{\mathcal{C}}$ with $\mathcal{C} \in \mathbb{R}_{>0}^{3}$.

We will see in Theorem 4.3 that the steady state locus in this system is 3 -dimensional. A forthcoming work will concern the question of how many times the steady state locus intersects the relative interior of a compatibility class $\mathcal{P}_{\mathcal{C}}$ for multisite phosphorylation systems 4 .

4.2. Results. For the $n$-site phosphorylation system, we will call its complex-to-species rate matrix $\Sigma_{n}$, and we will let $G_{n}$ denote the underlying digraph of the chemical reaction network. In order to apply the results of Section 3 to this system, we now aim to exhibit a specific basis of the kernel of $\Sigma_{n}$ that satisfies Condition 3.1. We begin by describing the rows of $\Sigma_{n}:=Y^{t} \cdot A_{\kappa}^{t}$ as linear combinations of the rows of $A_{\kappa}^{t}$. Recall that $A_{\kappa}$ is minus the Laplacian matrix of the 
associated digraph. Letting $R(i)$ represent the $i$-th row of $A_{\kappa}^{t}$, we have:

$$
\Sigma_{n}:=Y^{t} \cdot A_{\kappa}^{t}=\left[\begin{array}{c}
R(1)+R(2 n+2) \\
R(2)+R(2 n+3) \\
\vdots \\
R(n+1)+R(3 n+2) \\
\frac{R(n+2)}{\vdots} \\
R(2 n+1) \\
R(3 n+3) \\
\vdots \\
\frac{R(4 n+2)}{R(1)+R(2)+\cdots+R(n+1)} \\
R(2 n+2)+\cdots+R(3 n+2)
\end{array}\right] \in \mathbb{R}^{(3 n+3) \times(4 n+2)}
$$

Our next aim is to exhibit a submatrix of $\Sigma_{n}$ that shares the same kernel as $\Sigma_{n}$. The only relations that exist among the rows of $A_{\kappa}^{t}$ arise from the fact that the sum of the rows in each of the four blocks equals zero. Consequently, it is straightforward to check that

$$
\operatorname{rank}\left(\Sigma_{n}\right)=3 n \text {. }
$$

Moreover, if we delete any of the first $3 n+1$ rows and the last two rows of $\Sigma_{n}$, we obtain a new matrix that has maximal rank. As we are interested in describing the kernel of $\Sigma_{n}$, we will discard the first and the last two rows, and we will focus on the resulting submatrix. Furthermore, as the $(n+1)$-st and $(2 n+2)$-nd columns on $\Sigma_{n}$ are equal to zero, we already know that $e_{n+1}$ and $e_{2 n+2}$, the $(n+1)$-st and $(2 n+2)$-nd canonical basis vectors of $\mathbb{R}^{4 n+2}$, belong to $\operatorname{ker}\left(\Sigma_{n}\right)$. Hence we can now focus on an even smaller submatrix of $\Sigma_{n}$ obtained by deleting the first and the last two rows, and the $(n+1)$-st and $(2 n+2)$-nd columns. We will call this submatrix $\Sigma_{n}^{\prime}$, and we will denote by $C(j)$ the column of $\Sigma_{n}^{\prime}$ which corresponds to the $j$-th column of $\Sigma_{n}$ after deleting the first row and the last two (for example, $C(n+2)$ will represent the $(n+1)$-st column of $\Sigma_{n}^{\prime}$ ). Then, if we call $\Sigma_{n}^{\prime \prime}$ the submatrix of $\Sigma_{n}^{\prime}$ formed by its first $3 n$ columns, the system $\Sigma_{n}^{\prime} v=0$ is equivalent to the following one:

$$
\Sigma_{n}^{\prime \prime}\left[\begin{array}{c}
v_{1} \\
\vdots \\
v_{3 n}
\end{array}\right]=-\left[\begin{array}{lll}
C(3 n+3) & \ldots & C(4 n+2)
\end{array}\right]\left[\begin{array}{c}
v_{3 n+1} \\
\vdots \\
v_{4 n}
\end{array}\right] .
$$

Let us call

$$
D:=\operatorname{det}\left(\Sigma_{n}^{\prime \prime}\right) \text {. }
$$

If $D \neq 0$, then we can use Cramer's rule to solve system (4.3). In fact, we will show in Proposition 4.4 that this is the case and that we can find solutions to the system $\Sigma_{n} w=0$ such that all the nonzero entries have the same sign.

Next we introduce a partition and a set of basis vectors $b^{i}$ that will be used to show that the $n$-site system satisfies Condition 3.1. The partition $I_{1}, I_{2}, \ldots, I_{n+2}$ of $\{1,2, \ldots, 4 n+2\}$ is the following:

$$
I_{j}=\{j, n+j+1,2 n+j+2,3 n+j+2\}(\text { for } 1 \leq j \leq n), \quad I_{n+1}=\{n+1\}, \quad I_{n+2}=\{2 n+2\} .
$$

The entries in our vectors $b^{i}$ will be certain determinants. More precisely, let $D_{\ell(j)}$ be minus the determinant of the matrix obtained by replacing $C(\ell(j))$ by $C(3 n+j+2)$ in $\Sigma_{n}^{\prime \prime}$, for $\ell(j)=$ $j, n+j+1,2 n+j+2$, where $1 \leq j \leq n$ :

$$
D_{\ell(j)}=-\operatorname{det}\left(\left[\begin{array}{c}
\stackrel{\ell(j)}{\downarrow} \\
C(1)|\ldots| C(3 n+j+2)|\ldots| C(3 n+2)]
\end{array}\right]\right) .
$$


Note that $D, D_{j}, D_{n+j+1}$, and $D_{2 n+j+2}$, for $1 \leq j \leq n$, define polynomial functions of $\kappa$ on $\mathbb{R}_{>0}^{6 n}$. We will show in Proposition 4.4 that these functions $D, D_{j}, D_{n+j+1}$, and $D_{2 n+j+2}$ are nonzero and have the same sign, for $1 \leq j \leq n$.

Now we may define the vectors $b^{1}, b^{2}, \ldots, b^{n}$ of $\mathbb{R}_{>0}^{4 n+2}$ by:

$$
\left(b^{j}\right)_{i}= \begin{cases}D_{j} & \text { if } i=j \\ D_{n+j+1} & \text { if } i=n+j+1 \\ D_{2 n+j+2} & \text { if } i=2 n+j+2 \\ D & \text { if } i=3 n+j+2 \\ 0 & \text { otherwise }\end{cases}
$$

for $1 \leq i \leq 4 n+2$, where $1 \leq j \leq n$.

We are now equipped to state our main result in this section.

Theorem 4.3. The $n$-site phosphorylation system has toric steady states. The steady state locus has dimension 3 and can be parametrized by

$$
\begin{aligned}
\mathbb{R}^{3} \rightarrow & \mathbb{R}^{3 n+3} \\
\left(t_{1}, t_{2}, t_{3}\right) \mapsto & \left(t_{3}, \frac{D_{2 n+3}}{D_{1}} t_{1} t_{3}, \ldots, \frac{D_{2 n+3}}{D_{1}} \ldots \frac{D_{3 n+2}}{D_{n}} t_{1}^{n} t_{3}, \frac{D_{n+2}}{D_{1}} t_{1} t_{2} t_{3}, \ldots, \frac{D_{n+2}}{D_{1}} \ldots \frac{D_{2 n+1}}{D_{n}} t_{1}^{n} t_{2} t_{3},\right. \\
& \left.\frac{D}{D_{1}} t_{1} t_{2} t_{3}, \ldots, \frac{D}{D_{n}} \frac{D_{2 n+3}}{D_{1}} \ldots \frac{D_{3 n+1}}{D_{n-1}} t_{1}^{n} t_{2} t_{3}, t_{1} t_{2}, t_{2}\right) .
\end{aligned}
$$

Moreover, the system satisfies Condition 3.1 with the partition $I_{1}, I_{2}, \ldots, I_{n+2}$ described in (4.5) and the basis $\left\{b^{1}, \ldots, b^{n}\right\} \cup\left\{e_{n+1}, e_{2 n+2}\right\}$ where the vectors $b^{j}$ are defined in (4.7) and $e_{n+1}$ and $e_{2 n+2}$ are the $(n+1)$-st and $(2 n+2)$-nd vectors of the canonical basis of $\mathbb{R}^{4 n+2}$. In addition, it satisfies Conditions 3.4 and 3.6 .

In particular,

$\tilde{x}=\left(1, \frac{D_{2 n+3}}{D_{1}}, \ldots, \frac{D_{2 n+3}}{D_{1}} \ldots \frac{D_{3 n+2}}{D_{n}}, \frac{D_{n+2}}{D_{1}}, \ldots, \frac{D_{n+2}}{D_{1}} \ldots \frac{D_{2 n+1}}{D_{n}}, \frac{D}{D_{1}}, \ldots, \frac{D}{D_{n}} \frac{D_{2 n+3}}{D_{1}} \ldots \frac{D_{3 n+1}}{D_{n-1}}, 1,1\right)$

is an explicit positive steady state of the system.

We remark that the parametrization given in the statement of this theorem, which is one of the possible parametrizations provided by Theorem 3.11, gives systematically what Wang and Sontag obtained "by hand" in [36]. We note that the fact that this variety (the steady state locus) has a rational parametrization is a special case of a rational parametrization theorem for general multisite post-translational modification systems as analyzed by Thomson and Gunawardena [34.

4.3. Proof of Theorem 4.3. We start with the following proposition:

Proposition 4.4. Let $D$ be the determinant defined in (4.4), and let $D_{j}, D_{n+j+1}$, and $D_{2 n+j+2}$ be as in (4.6), for $1 \leq j \leq n$. Then each polynomial function $D, D_{j}, D_{n+j+1}, D_{2 n+j+2}: \mathbb{R}_{>0}^{6 n} \rightarrow \mathbb{R}$ for $1 \leq j \leq n$, never vanishes, and these functions all have the same constant sign on $\mathbb{R}_{>0}^{6 n}$.

Proof. For this proof, we will denote by $R(i)$ the $i$-th row of the matrix obtained from $A_{\kappa}^{t}$ after deleting columns $n+1$ and $2 n+2$. (Note that this notation differs slightly from that introduced in equation (4.2).) The proof has two steps: first we demonstrate that $D \neq 0$ on the positive orthant, and then we show that the other functions $D_{j}, D_{n+j+1}$, and $D_{2 n+j+2}$ are also nonzero on the positive orthant and that their signs coincide with that of $D$. 
To prove that $D \neq 0$ on $\mathbb{R}_{>0}^{6 n}$, we proceed by induction on $n$. First, if $n=1$, we have:

$$
\Sigma_{1}^{\prime \prime}=\left[\begin{array}{ccc}
0 & k_{\mathrm{cat}_{0}} & -l_{\mathrm{on}_{0}} \\
k_{\mathrm{on}_{0}} & -k_{\mathrm{off}_{0}}-k_{\mathrm{cat}_{0}} & 0 \\
0 & 0 & l_{\mathrm{on}_{0}}
\end{array}\right]
$$

In this case, $D=-k_{\mathrm{on}_{0}} k_{\mathrm{cat}_{0}} l_{\mathrm{on}_{0}} \neq 0$, as we wanted.

For the $n>1$ case, we suppose now that the $D \neq 0$ result is valid for $G_{n-1}$, the network of the $(n-1)$-site phosphorylation system. In order to visualize the calculations, we will reorder the rows and columns of $\Sigma_{n}^{\prime \prime}$, placing $C(1), C(n+2)$, and $C(2 n+3)$ as the leftmost columns, and $R(2)+R(2 n+3), R(n+2)$, and $R(3 n+3)$ as the uppermost rows. We notice that this ordering does not alter the sign of the determinants, hence we can write

$$
D=\operatorname{det}\left(\left[\begin{array}{ccc|c}
0 & k_{\mathrm{cat}_{0}} & -l_{\mathrm{on}_{0}} & \cdots \\
k_{\mathrm{on}_{0}} & -k_{\mathrm{off}_{0}}-k_{\mathrm{cat}_{0}} & 0 & \mathbf{0} \\
0 & 0 & l_{\mathrm{on}_{0}} & \mathbf{0} \\
\hline \mathbf{0} & \mathbf{0} & \mathbf{0} & B
\end{array}\right]\right)=-k_{\mathrm{on}_{0}} k_{\mathrm{cat}_{0}} l_{\mathrm{on}_{0}} \operatorname{det}(B)
$$

where $B$ is a $(3 n-3) \times(3 n-3)$-submatrix of $\Sigma_{n}^{\prime \prime}$. This matrix $B$ does not include either $C(1), C(n+$ 2), $C(2 n+3)$, nor the first $(n+1)$-st or $(2 n+1)$-st rows of $\Sigma_{n}^{\prime \prime}$. We next will see how the matrix $B$ can be interpreted as the $3(n-1) \times 3(n-1)$-matrix $\Sigma_{n-1}^{\prime \prime}$, the corresponding matrix of the smaller network $G_{n-1}$. This interpretation will allow us to conclude by the inductive hypothesis that $D \neq 0$ in the positive orthant.

For the purpose of interpreting this submatrix of $\Sigma_{n}^{\prime \prime}$ as the matrix of $G_{n-1}$, it is important to note that the deletion of $C(1), C(n+2)$, and $C(2 n+3)$ from $\Sigma_{n}^{\prime \prime}$ is equivalent to calculating $\Sigma_{n}^{\prime \prime}$ after having deleted these columns from $A_{\kappa}^{t}$ before calculating $\Sigma_{n}$. In turn, it is also equivalent to having deleted all the reactions that begin at the first, $(n+2)$-nd and $(2 n+3)$-rd complexes of the network. Once we have additionally deleted the first, $(n+1)$-st, and $(2 n+1)$-st rows (i.e. $R(2)+R(2 n+3), R(n+2)$, and $R(3 n+3))$, we obtain a new submatrix of $\Sigma_{n}$ whose entries we can rename as follows:

$$
k_{\mathrm{on}_{\mathrm{j}}}=: k_{\mathrm{on}_{\mathrm{j}-1}}^{\prime}, \quad k_{\mathrm{off}_{\mathrm{j}}}=: k_{\mathrm{off}_{\mathrm{j}-1}}^{\prime}, \quad k_{\mathrm{cat}_{\mathrm{j}}}=: k_{\mathrm{cat}_{\mathrm{j}-1}}^{\prime}, l_{\mathrm{on}_{\mathrm{j}}}=: l_{\mathrm{on}_{\mathrm{j}-1}}^{\prime}, l_{\mathrm{off}_{\mathrm{j}}}=: l_{\mathrm{off}_{\mathrm{j}-1}}^{\prime}, l_{\mathrm{cat}_{\mathrm{j}}}=: l_{\mathrm{cat}_{\mathrm{j}-1}}^{\prime}
$$

In fact, this new matrix is the corresponding complex-to-species rate matrix $\Sigma_{n-1}^{\prime}$ for the network $G_{n-1}$, with corresponding rate constants indicated by primes. We can also establish a correspondence between the nodes of the two networks: letting $j^{\prime}$ denote the $j$-th node of $G_{n-1}$, then $j^{\prime}$ corresponds to the following node of $G_{n}$ :

$j^{\prime}$ corresponds to $\begin{cases}j+1 & \text { if } 1 \leq j^{\prime} \leq n \quad\left(\text { complexes } S_{0}+E, \ldots, S_{n-1}+E \text { in } G_{n-1}\right) \\ j+2 & \text { if } n+1 \leq j^{\prime} \leq 2 n \quad\left(\text { complexes } E S_{0}, \ldots, E S_{n-2} \text { in } G_{n-1}\right) \\ j+3 & \text { if } 2 n+1 \leq j^{\prime} \leq 3 n-1 \quad\left(\text { complexes } S_{0}+F, \ldots, S_{n-1}+F \text { in } G_{n-1}\right) \\ j+4 & \text { if } 3 n \leq j^{\prime} \leq 4 n-2 \quad\left(\text { complexes } F S_{0}, \ldots, F S_{n-1} \text { in } G_{n-1}\right) .\end{cases}$

From this correspondence, it follows that $\operatorname{det}(B)$ equals $\operatorname{det}\left(\Sigma_{n-1}^{\prime \prime}\right)$, which is nonzero by inductive hypothesis, and therefore $D \neq 0$, which we wanted to prove.

We now complete the proof by verifying the following claim: the polynomial functions $D_{j}$, $D_{n+j+1}, D_{2 n+j+2}$ never vanish, and they all have the same constant sign as that of $D$ on $\mathbb{R}_{>0}^{6 n}$ (for $1 \leq j \leq n)$. 
We first prove this claim for the case $j=1$. We again reorder the entries of the matrices as described above, and as this ordering does not alter the sign of the determinants, we can write:

$$
\begin{aligned}
& D_{1}=-\operatorname{det}\left(\left[\begin{array}{ccc|c}
l_{\mathrm{off}_{0}} & k_{\mathrm{cat}_{0}} & -l_{\mathrm{on}_{0}} & \cdots \\
0 & -k_{\mathrm{off}_{0}}-k_{\mathrm{cat}_{0}} & 0 & \mathbf{0} \\
-l_{\mathrm{cat}_{0}}-l_{\mathrm{off}_{0}} & 0 & l_{\mathrm{on}_{0}} & \mathbf{0} \\
\hline \mathbf{0} & \mathbf{0} & \mathbf{0} & B
\end{array}\right]\right)=-\left(k_{\mathrm{off}_{0}}+k_{\mathrm{cat}_{0}}\right) l_{\mathrm{on}_{0}} l_{\mathrm{cat}_{0}} \operatorname{det}(B), \\
& D_{n+2}=-\operatorname{det}\left(\left[\begin{array}{ccc|c|c}
0 & l_{\mathrm{off}_{0}} & -l_{\mathrm{on}_{0}} & \cdots \\
k_{\mathrm{on}_{0}} & 0 & 0 & \mathbf{0} \\
0 & -l_{\mathrm{cat}_{0}}-l_{\mathrm{off}_{0}} & l_{\mathrm{on}_{0}} & \mathbf{0} \\
\hline \mathbf{0} & \mathbf{0} & \mathbf{0} & B
\end{array}\right]\right)=-k_{\mathrm{on}_{0}} l_{\mathrm{on}_{0}} l_{\mathrm{cat}_{0}} \operatorname{det}(B), \\
& D_{2 n+3}=-\operatorname{det}\left(\left[\begin{array}{cccc|c}
0 & k_{\mathrm{cat}_{0}} & l_{\mathrm{off}_{0}} & \mathbf{0} \\
k_{\mathrm{on}_{0}} & -k_{\mathrm{off}_{0}}-k_{\mathrm{cat}_{0}} & 0 & 0 \\
0 & 0 & -l_{\mathrm{cat}_{0}}-l_{\mathrm{off}_{0}} & \mathbf{0} \\
\hline \mathbf{0} & \mathbf{0} & \mathbf{0} & B
\end{array}\right]\right)=-k_{\mathrm{on}_{0}} k_{\mathrm{cat}_{0}}\left(l_{\mathrm{cat}_{0}}+l_{\mathrm{off}_{0}}\right) \operatorname{det}(B),
\end{aligned}
$$

where $B$ is the same matrix we described in equation (4.8). That is, $B=\Sigma_{n-1}^{\prime \prime}$. As we already know that $D \neq 0$, we deduce that $\operatorname{det}(B) \neq 0$. By examining equation (4.8) and the display above, we conclude that the claim is true for $j=1$.

For the $j>1$ case, we will prove our claim by induction on $n$. The base case is $n=2$ (as $j>1$ is not possible when $n=1$ ). In this case, the functions of interest are the following positive functions on $\mathbb{R}_{>0}^{12}: D=k_{\mathrm{on}_{0}} k_{\mathrm{cat}_{0}} l_{\mathrm{on}_{0}} k_{\mathrm{on}_{1}} k_{\mathrm{cat}_{1}} l_{\mathrm{on}_{1}}, D_{2}=k_{\mathrm{on}_{0}} k_{\mathrm{cat}_{0}} l_{\mathrm{on}_{0}}\left(k_{\mathrm{off}_{1}}+k_{\mathrm{cat}_{1}}\right) l_{\mathrm{on}_{1}} l_{\mathrm{cat}_{1}}$, $D_{5}=k_{\mathrm{on}_{0}} k_{\mathrm{cat}_{0}} l_{\mathrm{on}_{0}} k_{\mathrm{on}_{1}} l_{\mathrm{on}_{1}} l_{\mathrm{cat}_{1}}$, and $D_{8}=k_{\mathrm{on}_{0}} k_{\mathrm{cat}_{0}} l_{\mathrm{on}_{0}} k_{\mathrm{on}_{1}} k_{\mathrm{cat}_{1}}\left(l_{\mathrm{cat}_{1}}+l_{\mathrm{off}_{1}}\right)$. Hence our claim holds for $n=2$.

We now assume that the claim is true for $G_{n-1}$. As we did above, we view $G_{n-1}$ as a subgraph of $G_{n}$, and if we call $D_{\ell\left(j^{\prime}\right)}^{\prime}$ the corresponding determinant of the $(n-1)$-site system (for $\ell\left(j^{\prime}\right)=$ $j^{\prime},(n-1)+j^{\prime}+1,2(n-1)+j^{\prime}+2$, for $\left.1 \leq j^{\prime} \leq n-1\right)$, then we have:

$$
D_{\ell(j)}=(-1)^{(n+1)+1} k_{\mathrm{on}_{0}}(-1)^{1+n} k_{\mathrm{cat}_{0}}(-1)^{(2 n-1)+(2 n-1)} l_{\mathrm{on}_{0}} D_{\ell\left(j^{\prime}\right)}^{\prime}=-k_{\mathrm{on}_{0}} k_{\mathrm{cat}_{0}} l_{\mathrm{on}_{0}} D_{\ell\left(j^{\prime}\right)}^{\prime},
$$

for $\ell\left(j^{\prime}\right)=j^{\prime},(n-1)+j^{\prime}+1,2(n-1)+j^{\prime}+2$, where $1 \leq j^{\prime} \leq n-1$. By the inductive hypothesis, the claim holds for the $D_{\ell\left(j^{\prime}\right)}^{\prime}$, so by equation (4.9), the claim holds for the $D_{\ell(j)}$ as well. This completes the proof.

We now take care of the zero entries of the vectors $b^{j}$ defined in (4.7). We start by defining $D_{u \leftrightarrow v}$ as minus the determinant of the matrix obtained by replacing column $C(u)$ by $C(v)$ in $\Sigma_{n}^{\prime \prime}$, for $1 \leq u \leq 3 n+2$ such that $u \neq n+1, u \neq 2 n+2$, and $3 n+3 \leq v \leq 4 n+2$ :

$$
D_{u \leftrightarrow v}:=-\operatorname{det}\left(\left[C(1)|\ldots| C^{\grave{\downarrow}}(v)|\ldots| C(3 n+2)\right]\right) .
$$

We will deduce from the following lemma that $D_{u \leftrightarrow v}$ is equal to zero unless $u=j, n+j+1$, or $2 n+j+2$ and $v=3 n+j+2$, for $1 \leq j \leq n$.

Lemma 4.5. Fix $j \in\{1,2, \ldots, n\}$ and call $\widehat{\Sigma_{n}^{\prime}}$, the submatrix of $\Sigma_{n}^{\prime}$ obtained by deleting any two columns indexed by two elements of $I_{j}$. It holds that any $3 n \times 3 n$-minor of $\widehat{\Sigma_{n}^{\prime}}$ is equal to zero.

Proof. We will keep the notation $R(i)$ from the proof of Proposition 4.4. We now prove the lemma first for $j=1$, then $j=n$, and then finally for $1<j<n$.

For the case $j=1$, we focus on the reactions $1 \rightleftarrows n+2 \rightarrow 2,2 n+3 \rightleftarrows 3 n+3 \rightarrow 2 n+2$, and $3 n+4 \rightarrow 2 n+3$. If we delete $C(1)$ and $C(n+2)$, or $C(2 n+3)$ and $C(3 n+3)$, then the rows of $\widehat{\Sigma_{n}^{\prime}}$ corresponding to $R(n+2)$ or $R(3 n+3)$ will be equal to zero and the minor will be zero.

If we delete $C(1)$ and $C(2 n+3)$ (or $C(3 n+3))$, or we delete $C(n+2)$ and $C(2 n+3)$ (or $C(3 n+3))$, the rows corresponding to $R(n+2)$ and $R(3 n+3)$ will have only one entry different from zero and the determinant will be obviously zero if the column corresponding to any of this entries is not 
considered, or it will be the product of two constants and a $(3 n-2) \times(3 n-2)$-minor that does not include the columns $C(1), C(n+2), C(2 n+3), C(3 n+3)$ nor the rows $R(n+2), R(3 n+3)$.

It is important to notice that the columns of $A_{\kappa}^{t}$ carry the information of the reactions whose source (educt) is the corresponding complex, therefore, $C(\ell)$ carries the information of the reaction whose source is the $\ell$-th complex. As the only complexes that generate reactions whose product is the $(n+2)$-nd or $(3 n+3)$-rd complexes are the first and $(2 n+2)$ complexes, respectively, it follows that the columns that are being considered in this new $(3 n-2) \times(3 n-2)$-minor carry the information of reactions that do not end in either the $(n+2)$-nd or the $(3 n+3)$-rd complexes. Hence the sum of the rows in this new submatrix, and therefore the minor as well, is equal to zero.

For $j=n$, the analysis is similar.

For $1<j<n$ we focus on the reactions $j \rightleftarrows n+j+1 \rightarrow j+1$ and $2 n+j+2 \rightleftarrows 3 n+j+2 \rightarrow 2 n+j+1$. If we delete $C(j)$ and $C(n+j+1)$, or $C(2 n+j+2)$ and $C(3 n+j+2)$, then the rows of $\widehat{\Sigma_{n}^{\prime}}$ corresponding to $R(n+j+1)$ or $R(3 n+j+2)$ will be equal to zero and the minor will be zero.

If we delete $C(j)$ and $C(2 n+j+2)$ (or $C(3 n+j+2)$ ), or we delete $C(n+j+1)$ and $C(2 n+j+2)$ (or $C(3 n+j+2)$ ), the rows corresponding to $R(n+j+1)$ and $R(3 n+j+2)$ will have only one entry different from zero, and thus the determinant will be obviously zero if the column corresponding to any of these entries is not considered. Otherwise it will be the product of two nonzero rate constants and a $(3 n-2) \times(3 n-2)$-minor that does not include any of $C(j), C(n+j+1), C(2 n+$ $j+2), C(3 n+j+2)$ nor any of $R(n+j+1), R(3 n+j+2)$.

But deleting these columns is equivalent to not considering the reactions whose sources (educts) are the complexes $j, n+j+1,2 n+j+2$, or $3 n+j+2$. This disconnects the graph into four linkage classes, so this new graph gives a Laplacian matrix formed by four blocks. The rows of $\Sigma_{n}$ that we are considering in $\Sigma_{n}^{\prime}$ come from adding rows of the first and third blocks of $A_{\kappa}^{t}$, or the second and fourth ones; and the last rows of $\Sigma_{n}$, which correspond to intermediary species, clearly belong to only one of the blocks. Then, this new submatrix of $\widehat{\Sigma_{n}^{\prime}}$ can be reordered into a two-block matrix, for which the sums of the rows in each block are zero. Hence, the matrix obtained from $\widehat{\Sigma_{n}^{\prime}}$ without these four columns and two rows has rank at most $3 n-3$ and therefore any $(3 n-2) \times(3 n-2)$-minor will be zero.

We are now ready to prove Theorem 4.3 .

Proof of Theorem 4.3. Due to Lemma 4.5, for a $3 n \times 3 n$-minor of $\Sigma_{n}^{\prime}$ to be different from zero, we must obtain these $3 n$ columns by choosing three from each group indexed by $I_{j}$, for $1 \leq j \leq n$. In fact, any $3 n \times 3 n$-minor of $\Sigma_{n}^{\prime}$ that includes three columns from each group of four indexed by $I_{j}$, for $1 \leq j \leq n$, is always nonzero due to Proposition 4.4.

We can now solve system (4.3) by applying Cramer's rule. Recall the notation from (4.10):

$$
\left[\begin{array}{c}
v_{1} \\
\vdots \\
v_{3 n}
\end{array}\right]=\frac{-1}{D}\left[\begin{array}{ccc}
D_{1 \leftrightarrow 3 n+3} & \ldots & D_{1 \leftrightarrow 4 n+2} \\
\vdots & & \vdots \\
D_{3 n+2 \leftrightarrow 3 n+3} & \ldots & D_{3 n+2 \leftrightarrow 4 n+2}
\end{array}\right]\left[\begin{array}{c}
v_{3 n+1} \\
\vdots \\
v_{4 n}
\end{array}\right] .
$$

By Lemma 4.5, we already know that in the $3 n \times n$-matrix in the right-hand side above, the only nonzero entries are $D_{j}, D_{n+j+1}$, and $D_{2 n+j+2}$. This gives us a description of $\operatorname{ker}\left(\Sigma_{n}\right)$, which has a basis of the following form:

$$
\left\{e_{n+1}, e_{2 n+2}\right\} \cup\left\{b^{1}, b^{2}, \ldots, b^{n}\right\}
$$

for $b^{j}$ as in (4.7).

This proves that the $n$-site phosphorylation system satisfies Condition 3.1 for the partition $I_{1}, I_{2}, \ldots, I_{n+2}$ and the basis of $\operatorname{ker}\left(\Sigma_{n}\right),\left\{b^{1}, b^{2}, \ldots, b^{n}, e_{n+1}, e_{2 n+2}\right\}$, described above.

We now prove that the $n$-site phosphorylation system additionally satisfies Conditions 3.4 and 3.6. Condition 3.4 is satisfied immediately by Proposition 4.4. With respect to Condition 3.6, we notice 
that the subspace spanned by the columns of the matrix $\Delta$ has the following basis:

$$
\left\{e_{2 n+j+1}-e_{j}-e_{3 n+2}, e_{2 n+j+1}-e_{n+j+1}, e_{2 n+j+1}-e_{j+1}-e_{3 n+3} \mid 1 \leq j \leq n\right\} .
$$

Therefore, the dimension of the image of $\Delta$ is $3 n$, so $\operatorname{ker}(\Delta)=0$. Hence, equation (3.6) is trivially satisfied, as noted in Remark 3.7.

Then, by Theorem 3.8, it is immediate that the $n$-site phosphorylation system has toric steady states that are positive and real. Finally, for a parametrization of the steady state locus, let us consider the following matrix:

$$
A=\left[\begin{array}{ccccc|cccc|cccc|cc}
0 & 1 & 2 & \ldots & n & 1 & 2 & \ldots & n & 1 & 2 & \ldots & n & 1 & 0 \\
0 & 0 & 0 & \ldots & 0 & 1 & 1 & \ldots & 1 & 1 & 1 & \ldots & 1 & 1 & 1 \\
1 & 1 & 1 & \ldots & 1 & 1 & 1 & \ldots & 1 & 1 & 1 & \ldots & 1 & 0 & 0
\end{array}\right] \in \mathbb{R}^{3 \times(3 n+3)}
$$

It has maximal rank, and its kernel equals the span of all the differences $y_{j_{2}}-y_{j_{1}}$, for $j_{1}, j_{2} \in I_{j}$, where $1 \leq j \leq n+2$, shown in (4.11). After applying Theorem 3.11, we are left to see that the point $\tilde{x}$ defined in the statement of the present theorem is a positive steady state of the system. But it is easy to check that $\tilde{x}$ is a positive steady state by applying Theorem 3.3 to the following binomials:

$D x_{j} x_{3 n+2}-D_{j} x_{2 n+j+1}, D x_{n+j+1}-D_{n+j+1} x_{2 n+j+1}, D x_{j+1} x_{3 n+3}-D_{2 n+j+2} x_{2 n+j+1}$, for $1 \leq j \leq n$.

This completes the proof.

\section{MUltistationarity FOR SYSTEMS With TORIC STEADY STATES}

In this section we focus on the capacity of a chemical reaction system with toric steady states to exhibit multiple steady states. Following prior work of Conradi et al. [5] and Holstein [19], we make use of an alternative notation for reaction systems to obtain a characterization of steady states (Proposition 5.2). This result is used to prove a criterion for the existence of multistationarity for systems with toric steady states that satisfy Conditions [3.1, 3.4, and 3.6 (Theorem 5.5). At the end of this section, we make the connection to a related criterion of Feinberg.

Often a chemical reaction system has a continuum of steady states, as long as one steady state exists. However, as defined earlier (and as it is in Chemical Engineering), multistationarity refers to the existence of multiple steady states within one and the same stoichiometric compatibility class. In general one is interested in situations where the steady state locus intersects a stoichiometric compatibility class in a finite number of points [13. In Computational Biology one is sometimes interested in situations where the steady state locus intersects an affine subspace distinct from translates of the stoichiometric subspace $\mathcal{S}$ [15. Here we define multistationarity with respect to a linear subspace in the following way. Consider a matrix $Z \in \mathbb{R}^{s \times q}$, where $q$ is a positive integer. We say that the chemical reaction system $\dot{x}=\Sigma \cdot \Psi(x)$ exhibits multistationarity with respect to the linear subspace $\operatorname{ker}\left(Z^{t}\right)$ if and only if there exist at least two distinct positive steady state vectors $x^{1}, x^{2} \in \mathbb{R}_{>0}^{s}$ such that their difference lies in $\operatorname{ker}\left(Z^{t}\right)$; in other words the following equations must hold:

$$
\begin{aligned}
\Sigma \cdot \Psi\left(x^{1}\right) & =0 \\
\Sigma \cdot \Psi\left(x^{2}\right) & =0 \\
Z^{t} x^{1} & =Z^{t} x^{2} .
\end{aligned}
$$

Note that if the columns of $Z$ form a basis for $\mathcal{S}^{\perp}$, one recovers the usual definition of multistationarity given in Section 2.2. In this case, Equation (5.1c) states that the steady states $x^{1}$ and $x^{2}$ belong to the same stoichiometric compatibility class, and we simply speak of multistationarity, omitting the linear subspace we are referring to. 
5.1. Second representation of a chemical reaction system. We now introduce a second representation of the differential equations that govern a chemical reaction system (2.3); this will prove useful for the characterization of steady states (Proposition 5.2) and for establishing the capacity of a chemical reaction network for multistationarity. Letting $r$ denote the number of reactions of a chemical reaction network $G$, we fix an ordering of these $r$ reactions and define the incidence matrix $\mathcal{I} \in\{-1,0,1\}^{m \times r}$ of the network to be the matrix whose $i$-th column has a 1 in the row corresponding to the product complex of the $i$-th reaction and a -1 for the educt (reactant) complex. Then the $(s \times r)$-matrix product

$$
N:=Y^{t} \mathcal{I}
$$

is known as the stoichiometric matrix. Thus, the $i$-th column of $N$ is the reaction vector corresponding to reaction $i$. Next we define the educt-complex matrix

$$
\mathcal{Y}:=\left[\tilde{y}_{1}, \tilde{y}_{2}, \ldots, \tilde{y}_{r}\right],
$$

where the column $\tilde{y}_{i}$ of $\mathcal{Y}$ is defined as the vector of the educt complex of the $i$-th reaction. Now we can define the vector of educt complex monomials

$$
\phi(x):=\left(x^{\tilde{y}_{1}}, x^{\tilde{y}_{2}}, \ldots, x^{\tilde{y}_{r}}\right)^{t} .
$$

We also define $k \in \mathbb{R}_{>0}^{r}$ to be the vector of reaction rate constants: $k_{i}$ is the rate constant of the $i$-th reaction (that is, $k_{i}=\kappa_{i^{\prime} j^{\prime}}$ where the $i$-th reaction is from the complex $x^{y_{i^{\prime}}}$ to $x^{y_{j^{\prime}}}$ ). We now give a second formulation for a chemical reaction system (2.3) (cf. [16]):

$$
\dot{x}=N \operatorname{diag}(k) \phi(x) \text {. }
$$

Both formulations of a chemical reaction system given in equations (2.3) and (5.5) lead to the same system of ODEs and hence are equivalent. This can be made explicit by way of the doubling matrix $D$ of dimension $m \times r$ which relates $\mathcal{Y}$ and $Y$ via $\mathcal{Y}=Y^{t} D$. Here the $i$-th column vector of $D$ is defined as the unit vector $e_{j}$ of $\mathbb{R}^{m}$ such that $y_{j}$ is the educt (reactant) complex vector of the $i$-th reaction. From

$$
\dot{x}=N \operatorname{diag}(k) \phi(x)=Y^{t} \mathcal{I} \operatorname{diag}(k) D^{t} \Psi(x)=\Sigma \Psi(x),
$$

it follows that $\phi(x)=D^{t} \Psi(x)$ and $A_{\kappa}^{t}=\mathcal{I} \operatorname{diag}(k) D^{t}$.

Example 5.1. For the 1-site phosphorylation network (2.4), one obtains the matrices

$$
\begin{gathered}
\mathcal{I}=\left[\begin{array}{rrrrrr}
-1 & 1 & 0 & 0 & 0 & 0 \\
1 & -1 & -1 & 0 & 0 & 0 \\
0 & 0 & 1 & 0 & 0 & 0 \\
0 & 0 & 0 & -1 & 1 & 0 \\
0 & 0 & 0 & 1 & -1 & -1 \\
0 & 0 & 0 & 0 & 0 & 1
\end{array}\right], \quad D=\left[\begin{array}{llllll}
1 & 0 & 0 & 0 & 0 & 0 \\
0 & 0 & 0 & 0 & 0 & 0 \\
0 & 1 & 1 & 0 & 0 & 0 \\
0 & 0 & 0 & 0 & 0 & 0 \\
0 & 0 & 0 & 1 & 0 & 0 \\
0 & 0 & 0 & 0 & 1 & 1
\end{array}\right] \\
\mathcal{Y}=\left[y_{1}^{t}, y_{3}^{t}, y_{3}^{t}, y_{5}^{t}, y_{6}^{t}, y_{6}^{t}\right]=\left[\begin{array}{llllll}
1 & 0 & 0 & 0 & 0 & 0 \\
0 & 0 & 0 & 1 & 0 & 0 \\
0 & 1 & 1 & 0 & 0 & 0 \\
0 & 0 & 0 & 0 & 1 & 1 \\
1 & 0 & 0 & 0 & 0 & 0 \\
0 & 0 & 0 & 1 & 0 & 0
\end{array}\right]
\end{gathered}
$$

and the monomial vector $\phi(x)=\left(x_{1} x_{5}, x_{3}, x_{3}, x_{2} x_{6}, x_{4}, x_{4}\right)^{t}$.

It follows from the differential equations (5.5) that a positive concentration vector $x \in \mathbb{R}_{>0}^{s}$ is a steady state for the chemical reaction system defined by the positive reaction rate constant vector $k$ if and only if

$$
\operatorname{diag}(k) \phi(x) \in \operatorname{ker}(N) \cap \mathbb{R}_{>0}^{r} .
$$

We now recognize that the set $\operatorname{ker}(N) \cap \mathbb{R}_{>0}^{r}$, if nonempty, is the relative interior of the pointed polyhedral cone $\operatorname{ker}(N) \cap \mathbb{R}_{\geq 0}^{r}$. To utilize this cone, we collect a finite set of generators (also called 
"extreme rays") of the cone $\operatorname{ker}(N) \cap \mathbb{R}_{\geq 0}^{r}$ as columns of a non-negative matrix $M$. Up to scalar multiplication, generators of a cone are unique and form a finite set; as the cone of interest arises as the intersection of an orthant with a linear subspace, the generators are the vectors of the cone with minimal support with respect to inclusion. (Background on polyhedral cones can be found in the textbook of Rockafellar [27].) Letting $p$ denote the number of generators of the cone, we can use $M$ to express the condition for a positive vector $x \in \mathbb{R}_{>0}^{s}$ to be a steady state of the chemical reaction system in the following way:

$$
\operatorname{diag}(k) \phi(x)=M \lambda, \text { for some } \lambda \in \mathbb{R}_{\geq 0}^{p} \text { with } M \lambda \in \mathbb{R}_{>0}^{r} .
$$

Note that this proves the following result which appears in [5]:

Proposition 5.2 (Characterization of steady states of chemical reaction systems). For a chemical reaction network $G$, let $M$ denote a corresponding generator matrix as defined above. Then a positive vector $x \in \mathbb{R}_{>0}^{s}$ is a steady state for the chemical reaction system defined by reaction rate vector $k \in \mathbb{R}_{>0}^{r}$, if and only if there exists a vector $\lambda \in \mathbb{R}_{\geq 0}^{p}$ such that

$$
k=\operatorname{diag}\left((\phi(x))^{-1} M \lambda \text { and } M \lambda \in \mathbb{R}_{>0}^{r} .\right.
$$

We now note that outside of a degenerate case, any positive concentration vector can be a steady state for appropriately chosen rate constants $k$.

Remark 5.3. We now comment on the degenerate case of a network for which the set $\operatorname{ker}(N) \cap \mathbb{R}_{>0}^{r}$ is empty. First, this case is equivalent to either of the following three conditions: (i) there is no positive dependence among the reaction vectors $\left(y_{j}-y_{i}\right)$, (ii) the cone $\operatorname{ker}(N) \cap \mathbb{R}_{\geq 0}^{r}$ is contained in a coordinate hyperplane, and (iii) the generator matrix $M$ has at least one zero row. Now, in this degenerate case, it is clear that for any choice of reaction rate constants, the chemical reaction system has no positive steady states. This is because if $x^{*} \in \mathbb{R}_{>0}^{s}$ is a steady state for the system with reaction rate constants $\kappa_{i j}$, then the numbers $\alpha_{i j}:=\kappa_{i j} \cdot\left(x^{*}\right)^{y_{i}}$ witness to the positive dependence among the reaction vectors $\left(y_{j}-y_{i}\right)$ 's. Outside of this degenerate case, it follows from Proposition 5.2 that there exists a vector of reaction rate constants $k$ for which the resulting chemical reaction system has a positive steady state. Moreover, in this case any positive vector $x$ can be a steady state, by choosing $k$ as in equation (5.7) for some valid choice of $\lambda \in \mathbb{R}_{\geq 0}^{p}$.

Using our new notation, we return to the question of existence of steady states.

Remark 5.4. Recall the content of Corollary 3.12, for a chemical reaction network for which a single partition works to satisfy Condition 3.1 for all choices of positive rate constants, the set of rate constant vectors $k$ that yield systems with positive steady states is the semialgebraic set of $\mathbb{R}_{>0}^{r}$ defined by Conditions 3.4 and 3.6. We now note that Proposition 5.2 implies that this set of rate constant vectors is the image of the following polynomial map:

$$
\begin{aligned}
\beta: \quad \mathbb{R}_{>0}^{s} \times \Gamma & \rightarrow \mathbb{R}_{>0}^{r} \\
(x, \lambda) & \mapsto \operatorname{diag}(\phi(x))^{-1} M \lambda,
\end{aligned}
$$

where $\Gamma:=\left\{\lambda \in \mathbb{R}_{>0}^{p} \mid M \lambda \in \mathbb{R}_{>0}^{r}\right\}$. In case that Condition 3.1 holds and Condition 3.6 is trivially satisfied (i.e. $\Delta$ has full row rank), the image of $\beta$ is cut out by the inequalities defined by Condition 3.4.

5.2. Main result on multistationarity. We now make use of Proposition 5.2 to examine which chemical reaction systems with toric steady states exhibit multistationarity. We first note that in the setting of Section 3, the set of differences $\ln x^{1}-\ln x^{2}$, where $x^{1}$ and $x^{2}$ are positive steady states for the same system, form a linear subspace. As before, the notation "ln $x$ " for a vector $x \in \mathbb{R}_{>0}^{s}$ denotes the vector $\left(\ln x_{1}, \ln x_{2}, \ldots, \ln x_{s}\right) \in \mathbb{R}^{s} ;$ similarly we will make use of the notation " $e$ " to denote component-wise exponentiation. 
Our next theorem, the main result of this section, is a consequence of [5, Lemma 1]. It states that a network that satisfies Condition 3.1 has the capacity for multistationarity if and only if two subspaces, namely $\operatorname{im}\left(A^{t}\right)$ and $\mathcal{S}$, both intersect non-trivially some (possibly lower-dimensional) orthant $\left\{x \in \mathbb{R}^{s} \mid \operatorname{sign}(x)=\omega\right\}$ defined by a sign vector $\omega \in\{-, 0,+\}^{s}$. We remark that this is a matroidal condition. Related ideas appear in work of Feinberg [14], and details on the connection between our work and Feinberg's appears at the end of this section.

Theorem 5.5 (Multistationarity for networks with toric steady states). Fix a chemical reaction network $G$ with s species and $m$ complexes, and let $Z \in \mathbb{Z}^{s \times q}$ be an integer matrix, for some positive integer $q$. Assume that the cone $\operatorname{ker}(N) \cap \mathbb{R}_{>0}^{r}$ is not contained in any coordinate hyperplane. Assume moreover that there exists a partition $I_{1}, I_{2}, \ldots, I_{d}$ of the $m$ complexes of $G$ such that Condition 3.1 is satisfied for all rate constants.

Recall the matrix A for this partition from the proof of Theorem 3.11. Then there exists a reaction rate constant vector such that the resulting chemical reaction system exhibits multistationarity with respect to the linear subspace $\operatorname{ker}\left(Z^{t}\right)$ if and only if there exists an orthant of $\mathbb{R}^{s}$ that both subspaces $\operatorname{im}\left(A^{t}\right)$ and $\operatorname{ker}\left(Z^{t}\right)$ intersect nontrivially. More precisely, given nonzero vectors $\alpha \in \operatorname{im}\left(A^{t}\right)$ and $\sigma \in \operatorname{ker}\left(Z^{t}\right)$ with

$$
\operatorname{sign}(\alpha)=\operatorname{sign}(\sigma),
$$

then two steady states $x^{1}$ and $x^{2}$ and a reaction rate constant vector $k$ that witness multistationarity (that is, that satisfy equations (5.1a), (5.1b), and (5.1c)) arise in the following way:

$$
\left(x_{i}^{1}\right)_{i=1, \ldots, s}=\left\{\begin{array}{l}
\frac{\sigma_{i}}{e^{\alpha_{i}}-1}, \text { if } \alpha_{i} \neq 0 \\
\bar{x}_{i}>0, \text { if } \alpha_{i}=0,
\end{array}\right.
$$

where $\bar{x}_{i}$ denotes an arbitrary positive number, and

$$
\begin{aligned}
x^{2} & =\operatorname{diag}\left(e^{\alpha}\right) x^{1} \\
k & =\operatorname{diag}\left(\phi\left(x^{1}\right)\right)^{-1} M \lambda,
\end{aligned}
$$

for any non-negative vector $\lambda \in \mathbb{R}_{>0}^{p}$ for which $M \lambda \in \mathbb{R}_{>0}^{r}$. Conversely, any witness to multistationarity with respect to $\operatorname{ker}\left(Z^{\bar{t}}\right)$ (given by some $x^{1}, x^{2} \in \mathbb{R}_{>0}^{s}$, and $k \in \mathbb{R}_{>0}^{r}$ ) arises from equations (5.8), (5.9), (5.10), and (5.11) for some vectors $\alpha \in \operatorname{im}\left(A^{t}\right)$ and $\sigma \in \operatorname{ker}\left(Z^{t}\right)$ that have the same sign.

Proof. Assume that there exist nonzero vectors $\alpha \in \operatorname{im}\left(A^{t}\right)$ and $\sigma \in \operatorname{ker}\left(Z^{t}\right)$ having the same sign. First note that the vectors $x^{1}, x^{2}$, and $k$ defined by (5.9), (5.10), and (5.11), respectively, are positive because $\alpha$ and $\sigma$ have the same sign and because the cone $\operatorname{ker}(N) \cap \mathbb{R}_{\geq 0}^{r}$ is not contained in a coordinate hyperplane. By Proposition [5.2, equation (5.11) implies that $x^{1}$ is a steady state of the system defined by $k$. We now claim that $x^{2}$ too is a steady state of the same system. This follows from Theorem 3.11 because the difference between $\ln x^{1}$ and $\ln x^{2}$ is in $\operatorname{im}\left(A^{t}\right)$ :

$$
\ln x^{1}-\ln x^{2}=-\alpha \in \operatorname{im}\left(A^{t}\right) .
$$

Conversely, assume that vectors $x^{1}, x^{2}$, and $k$ are a witness to multistationarity with respect to $\operatorname{ker}\left(Z^{t}\right)$. Let us now construct appropriate vectors $\alpha$ and $\sigma$. By Theorem 3.11, the vector $\alpha:=\ln x^{2}-\ln x^{1}$ is in $\operatorname{im}\left(A^{t}\right)$. Next, we define $\sigma \in \mathbb{R}^{s}$ by $\sigma_{i}=\left(e^{\alpha_{i}}-1\right) x_{i}^{1}$ if $\alpha_{i} \neq 0$ and $\sigma_{i}=0$ if $\alpha_{i}=0$, so by construction, $\alpha$ and $\sigma$ have the same sign. In addition, equations (5.9) and (5.10) easily follow for these values of $\alpha$ and $\sigma$. We also see that

$$
-\sigma=x^{1}-x^{2} \in \operatorname{ker}\left(Z^{t}\right),
$$

so $\sigma \in \operatorname{ker}\left(Z^{t}\right)$. Finally, Proposition 5.2 implies that there exists a valid $\lambda \in \mathbb{R}_{\geq 0}^{p}$ that satisfies (5.11). 
Remark 5.6. If a chemical reaction system defined by reaction rate constants $k^{*}$ and a partition of its complexes satisfy Conditions 3.1, 3.4, and 3.6 (but not necessarily for other choices of rate constants), then the equations (5.8), (5.9), (5.10), and (5.11) in Theorem 5.5) still characterize multistationarity. In other words, $x^{1}$ and $x^{2}$ are two steady states that demonstrate that the system defined by $k^{*}$ has the capacity for multistationarity with respect to $\operatorname{ker}\left(Z^{t}\right)$ if and only if there exist $\alpha \in \operatorname{im}\left(A^{t}\right), \sigma \in \operatorname{ker}\left(Z^{t}\right)$, and $\lambda \in \mathbb{R}_{\geq 0}^{p}$ such that those four equations hold.

Example 5.7 (Triangle network, continued). We return to the Triangle network analyzed in Examples 2.3 and 3.10. The stoichiometric subspace is

$$
\operatorname{ker}(\Sigma)=\mathcal{S}=\operatorname{span}\{(1,-1)\}
$$

In the toric setting (recall that this is when $\kappa_{31}=\kappa_{32}$ ), the partition for which the system satisfies Condition 3.1 is $\{1,2\},\{3\}$, so a matrix A for which

$$
\operatorname{ker}(A)=\operatorname{span}\left\{y_{2}-y_{1}\right\}=\operatorname{span}\{(2,-2)\}
$$

is $A=\left[\begin{array}{ll}1 & 1\end{array}\right]$. We can see that the subspaces $\operatorname{ker}\left(Z^{t}\right)$ and $\operatorname{im}\left(A^{t}\right)=\operatorname{span}\{(1,1)\}$ do not both intersect some orthant nontrivially. So Theorem 5.5 allows us to conclude that no system (for which $\kappa_{31}=\kappa_{32}$ ) arising from the Triangle network exhibits multistationarity.

Although the capacity of the Triangle network to exhibit multistationarity is easily determined directly, without the need to apply Theorem 5.5, it is more difficult in the case of the multisite phosphorylation system. Recall that we proved in Theorem 4.3 that any $n$-site phosphorylation system satisfies Condition [3.1 with the same partition (for fixed $n$ ). Hence, Theorem [5.5 can be used to compute the semialgebraic set of reaction rate constants $k$ that give rise to multistationarity for the phosphorylation networks. This was performed by Conradi et al. (for the 2-site network) [5] and Holstein (for the general $n$-site network) [19]; multistationarity is possible only for $n \geq 2$. Results on the number of steady states of phosphorylation systems appeared in work of Wang and Sontag [36] and is the focus of a forthcoming work of the authors [4].

5.3. Connection to related results on multistationarity. We now make the connection between our results on the capacity of a chemical reaction network to exhibit multistationarity and related results of Feinberg [14. A regular network is a network for which (i) $\operatorname{ker}(N) \cap \mathbb{R}_{>0}^{r} \neq \emptyset$, (ii) each linkage class contains a unique terminal strong linkage class, and (iii) removing the reaction(s) between any two adjacent complexes in a terminal strong linkage class disconnects the corresponding linkage class. Recall from Remark 5.3 that condition (i) in this definition is simply the requirement that the reaction vectors $y_{j}-y_{i}$ are positively dependent, and that this condition is necessary for the existence of positive steady states. Recall that the deficiency of a chemical reaction network was discussed in $\S 2.3$.

We now can explain the relationship between Feinberg's result and ours. Feinberg examined regular deficiency-one networks, while we are concerned with networks for which there exists a partition that satisfies Condition 3.6 (for all rate constants). In these respective settings, both Theorem 4.1 and Corollary 4.1 of [14] and Theorem 5.5] in this article state that a certain subset of $\mathbb{R}^{s}$ and the stoichiometric subspace both intersect the same orthant non-trivially if and only if the network has the capacity for multistationarity. In the result of Feinberg, this set is a union of certain polyhedral cones, while in our case, this set is the image of $A^{t}$. In both cases, this set consists of all vectors $\ln \left(c^{*} / c^{* *}\right)$, where $c^{*}$ and $c^{* *}$ are steady states arising from the same rate constants. As an illustration, see Example 5.8 below.

Let us now explain how the two results are complementary. First, there are some networks for which only Feinberg's results apply. For example, consider any network for which the union of polyhedral cones obtained from Feinberg's results is not a linear space. Additionally, for some networks, only our results apply. As an example, the $n>1$ multisite networks have deficiency 
greater than one. Finally, for some networks, both our results and Feinberg's apply, such as in the following example.

Example 5.8. The 1-site phosphorylation network of Example 2.1 is regular and has deficiency one. In this case, both the image of $A^{t}$ and Feinberg's union of cones are the subspace of $\mathbb{R}^{6}$ spanned by the three vectors $\left(e_{1}+e_{2}+e_{3}+e_{4}\right),\left(e_{2}+e_{3}+e_{4}+e_{5}\right)$, and $\left(e_{3}+e_{4}+e_{5}+e_{6}\right)$. So in this instance, our Theorem 5.5 and Feinberg's Corollary 4.1 of [14] coincide.

Finally we note that the proofs of both results make use of special structure of $\operatorname{ker}(\Sigma)$. In our case, we assume the existence of a basis with disjoint support. For Feinberg's results, there is a non-negative basis where the supports of the first $L$ basis vectors correspond exactly to the $L$ terminal strong linkage classes, and the last basis vector is the all-ones vector (here $L$ denotes the number of terminal strong linkage classes).

Acknowledgments: We thank the Statistical and Applied Mathematical Sciences Institute (SAMSI), USA, where this work was started. We are grateful to the organizers of the 2008-09 Program on Algebraic Methods in Systems Biology and Statistics at SAMSI, for generating the space for our interactions. We acknowledge two conscientious referees whose comments improved this article.

\section{REFERENCES}

[1] D. Angeli, P. De Leenheer, and E. Sontag, A Petri net approach to persistence analysis in chemical reaction networks, Biology and Control Theory: Current Challenges (Isabelle Queinnec, Sophie Tarbouriech, Germain Garcia, and Silviu-Iulian Niculescu, eds.), Lecture Notes in Control and Information Sciences, vol. 357, Springer Berlin/Heidelberg, 2007, pp. 181-216.

[2] D. Battogtokh and J. J. Tyson, (2004), Bifurcation analysis of a model of the budding yeast cell cycle, Chaos $14(3), 653-661$.

[3] K. C. Chen, L. Calzone, A. Csikasz-Nagy, F. R. Cross, B. Novak, and J. J. Tyson, (2004), Integrative analysis of cell cycle control in budding yeast, Mol. Biol. Cell 15(8), 3841-3862.

[4] C. Conradi, A. Dickenstein, M. Pérez Millán, and A. Shiu, Counting positive roots of polynomials with applications for biochemical systems, In preparation.

[5] C. Conradi, D. Flockerzi, and J. Raisch, (2008), Multistationarity in the activation of a MAPK: Parametrizing the relevant region in parameter space, Math. Biosci. 211(1), 105-131.

[6] C. Conradi, J. Saez-Rodriguez, E.-D. Gilles, and J. Raisch, (2005), Using Chemical Reaction Network Theory to discard a kinetic mechanism hypothesis, IEE Proc. Systems Biology (now IET Systems Biology) 152(4), 243-248.

[7] D. Cox, J. Little, and D. O'Shea, (1992) Ideals, varieties, and algorithms: an introduction to computational algebraic geometry and commutative algebra, Springer-Verlag, New York.

[8] G. Craciun, A. Dickenstein, A. Shiu , and B. Sturmfels, (2009) Journal of Symbolic Computation 44, 1551-1565.

[9] R. J. Deshaies and J. E. Ferrell, (2001), Multisite phosphorylation and the countdown to S phase, Cell 107(7), 819-822.

[10] D. Eisenbud and B. Sturmfels, (1996), Binomial ideals, Duke Math. J. 84(1), 1-45.

[11] M. Feinberg, (1972), Complex balancing in general kinetic systems, Arch. Rational Mech. Anal. 49(3), $187-194$.

[12] M. Feinberg, (1989), Necessary and sufficient conditions for detailed balancing in mass action systems of arbitrary complexity, Chem. Eng. Sci. 44(9), 1819-1827.

[13] M. Feinberg, (1995), The existence and uniqueness of steady states for a class of chemical reaction networks, Arch. Rational Mech. Anal. 132(4), 311-370.

[14] M. Feinberg, (1995), Multiple steady states for chemical reaction networks of deficiency one, Arch. Rational Mech. Anal. 132(4), 371-406.

[15] D. Flockerzi and C. Conradi, (2008), Subnetwork analysis for multistationarity in mass-action kinetics, J. Phys. Conf. Ser. 138(1), 012006.

[16] K. Gatermann and B. Huber, (2002), A family of sparse polynomial systems arising in chemical reaction systems, J. Symbolic Comput. 33(3), 275-305.

[17] N. Hermann-Kleiter and G. Baier, (2010), NFAT pulls the strings during CD4+ T helper cell effector functions, Blood 115(15), 2989-2997.

[18] P. G. Hogan, L. Chen, J. Nardone, and A. Rao, (2003), Transcriptional regulation by calcium, calcineurin, and NFAT, Gene Dev. 17(18), 2205-2232. 
[19] K. Holstein, (2008), Mathematische analyse der $n$-fachen Phosphorylierung eines Proteins: Existenz mehrfach stationärer Zustände. Master's thesis, Diplomarbeit, Universität Magdeburg.

[20] F. Horn, (1972), Necessary and sufficient conditions for complex balancing in chemical kinetics, Arch. Ration. Mech. Anal. 49(3), 172-186.

[21] F. Horn and R. Jackson, (1972), General mass action kinetics, Arch. Ration. Mech. Anal. 47(2), 81-116.

[22] C.-Y. F. Huang and J. E. Ferrell, (1996), Ultrasensitivity in the Mitogen-Activated Protein Kinase Cascade, PNAS 93(19), 10078-10083.

[23] O. Kapuy, D. Barik, M. R.-D. Sananes, J. J. Tyson, and B. Novák, (2009), Bistability by multiple phosphorylation of regulatory proteins, Prog. Biophys. Mol. Bio. 100(1-3), 47-56.

[24] F. Macian, (2005), NFAT proteins: key regulators of T-cell development and function, Nat. Rev. Immunol. 5(6), $472-484$.

[25] A. K. Manrai and J. Gunawardena, (2008), The geometry of multisite phosphorylation, Biophys. J. 95(12), $5533-5543$.

[26] N. I. Markevich, J. B. Hoek, and B. N. Kholodenko, (2004), Signaling switches and bistability arising from multisite phosphorylation in protein kinase cascades, J. Cell Biol. 164(3), 353-359.

[27] R. T. Rockafellar (1970) Convex Analysis, Princeton University Press, Princeton NJ.

[28] W. Sha, J. Moore, K. Chen, A. D. Lassaletta, C.-S. Yi, J. J. Tyson, and J. C. Sible, (2003), Hysteresis drives cell-cycle transitions in xenopus laevis egg extracts, PNAS 100(3), 975-980.

[29] Y. D. Shaul and R. Seger, (2007), The MEK/ERK cascade: From signaling specificity to diverse functions, Biochim. Biophys. Acta. 1773(8), 1213-1226.

[30] G. Shinar and M. Feinberg, (2010), Structural sources of robustness in biochemical reaction networks, Science 327(5971), 1389-1391.

[31] G. Strang (1976) Linear Algebra and its Applications, Academic Press, New York.

[32] R. Thomas and M. Kaufman, (2001), Multistationarity, the basis of cell differentiation and memory. I. Structural conditions of multistationarity and other nontrivial behavior, Chaos 11(1), 170-179.

[33] R. Thomas and M. Kaufman, (2001), Multistationarity, the basis of cell differentiation and memory. II. Logical analysis of regulatory networks in terms of feedback circuits, Chaos 11(1), 180-195.

[34] M. Thomson and J. Gunawardena, (2009), The rational parameterisation theorem for multisite post-translational modification systems, J. Theor. Biol. 261(4), 626-636.

[35] M. Thomson and J. Gunawardena, (2009), Unlimited multistability in multisite phosphorylation systems, Nature $460(7252), 274-277$.

[36] L. Wang and E. Sontag, (2008), On the number of steady states in a multiple futile cycle, J. Math. Biol. 57(1), $29-52$.

MPM: Dto. de Matemática, FCen, Universidad de Buenos Aires, Ciudad Universitaria, Pab. I, C1428Ega Buenos Aires, Argentina. AD: Dto. de Matemática, FCEn, Universidad de Buenos Aires, Ciudad Universitaria, Pab. I, C1428EgA Buenos Aires, Argentina, and imas/COniCET. AS: Dept. of Mathematics, Duke University, Box 90320, Durham NC 27708-0320, USA. CC: MaX-Planck-Institut Dynamik komplexer technischer Systeme, Sandtorstr. 1, 39106 Magdeburg, Germany.

E-mail address: mpmillan@dm.uba.ar, alidick@dm.uba.ar, annejls@math.duke.edu, conradi@mpi-magdeburg.mpg.de 